\title{
The Gaia-ESO Survey: Sodium and aluminium abundances in giants and dwarfs
}

\section{Implications for stellar and Galactic chemical evolution $\star, \star \star$}

\author{
R. Smiljanic ${ }^{1}$, D. Romano ${ }^{2}$, A. Bragaglia ${ }^{2}$, P. Donati ${ }^{2,3}$, L. Magrini ${ }^{4}$, E. Friel ${ }^{5}$, H. Jacobson ${ }^{6}$, S. Randich $^{4}$, P. Ventura ${ }^{7}$, \\ K. Lind ${ }^{8,9}$, M. Bergemann ${ }^{9}$, T. Nordlander ${ }^{8}$, T. Morel ${ }^{10}$, E. Pancino ${ }^{2,11}$, G. Tautvaišiené ${ }^{12}$, V. Adibekyan $^{13}$, M. Tosi $^{2}$, \\ A. Vallenari ${ }^{14}$, G. Gilmore ${ }^{15}$, T. Bensby ${ }^{16}$, P. François ${ }^{17,18}$, S. Koposov ${ }^{15}$, A. C. Lanzafame ${ }^{19}$, A. Recio-Blanco $^{20}$, \\ A. Bayo $^{21}$, G. Carraro ${ }^{22}$, A. R. Casey ${ }^{15}$, M. T. Costado ${ }^{23}$, E. Franciosini ${ }^{4}$, U. Heiter ${ }^{24}$, V. Hill ${ }^{20}$, A. Hourihane ${ }^{15}$, \\ P. Jofré ${ }^{15}$, C. Lardo ${ }^{25}$, P. de Laverny ${ }^{20}$, J. Lewis ${ }^{15}$, L. Monaco ${ }^{26}$, L. Morbidelli ${ }^{4}$, G. .G. Sacco ${ }^{4}$, L. Sbordone ${ }^{27,28}$, \\ S. G. Sousa ${ }^{13}$, C. C. Worley ${ }^{15}$, and S. Zaggia ${ }^{14}$ \\ (Affiliations can be found after the references)
}

Received 20 December 2015 / Accepted 7 February 2016

\begin{abstract}
Context. Stellar evolution models predict that internal mixing should cause some sodium overabundance at the surface of red giants more massive than $\sim 1.5-2.0 M_{\odot}$. The surface aluminium abundance should not be affected. Nevertheless, observational results disagree about the presence and/or the degree of $\mathrm{Na}$ and $\mathrm{Al}$ overabundances. In addition, Galactic chemical evolution models adopting different stellar yields lead to very different predictions for the behavior of $[\mathrm{Na} / \mathrm{Fe}]$ and $[\mathrm{Al} / \mathrm{Fe}]$ versus $[\mathrm{Fe} / \mathrm{H}]$. Overall, the observed trends of these abundances with metallicity are not well reproduced.

Aims. We readdress both issues, using new $\mathrm{Na}$ and $\mathrm{Al}$ abundances determined within the Gaia-ESO Survey. Our aim is to obtain better observational constraints on the behavior of these elements using two samples: i) more than 600 dwarfs of the solar neighborhood and of open clusters and ii) low- and intermediate-mass clump giants in six open clusters.

Methods. Abundances were determined using high-resolution UVES spectra. The individual Na abundances were corrected for nonlocal thermodynamic equilibrium effects. For the Al abundances, the order of magnitude of the corrections was estimated for a few representative cases. For giants, the abundance trends with stellar mass are compared to stellar evolution models. For dwarfs, the abundance trends with metallicity and age are compared to detailed chemical evolution models.

Results. Abundances of $\mathrm{Na}$ in stars with mass below $\sim 2.0 M_{\odot}$, and of $\mathrm{Al}$ in stars below $\sim 3.0 M_{\odot}$, seem to be unaffected by internal mixing processes. For more massive stars, the Na overabundance increases with stellar mass. This trend agrees well with predictions of stellar evolutionary models. For Al, our only cluster with giants more massive than $3.0 M_{\odot}, \mathrm{NGC} 6705$, is $\mathrm{Al}$ enriched. However, this might be related to the environment where the cluster was formed. Chemical evolution models that well fit the observed $[\mathrm{Na} / \mathrm{Fe}] \mathrm{vs}$. $[\mathrm{Fe} / \mathrm{H}]$ trend in solar neighborhood dwarfs cannot simultaneously explain the run of $[\mathrm{Al} / \mathrm{Fe}]$ with $[\mathrm{Fe} / \mathrm{H}]$, and vice versa. The comparison with stellar ages is hampered by severe uncertainties. Indeed, reliable age estimates are available for only a half of the stars of the sample. We conclude that $\mathrm{Al}$ is underproduced by the models, except for stellar ages younger than about 7 Gyr. In addition, some significant source of late Na production seems to be missing in the models. Either current $\mathrm{Na}$ and $\mathrm{Al}$ yields are affected by large uncertainties, and/or some important Galactic source(s) of these elements has as yet not been taken into account.
\end{abstract}

Key words. Galaxy: abundances - Galaxy: evolution - stars: abundances - stars: evolution - stars: late-type

\section{Introduction}

Sodium and aluminium are two odd-Z elements with single stable isotopes $\left({ }^{23} \mathrm{Na}\right.$ and ${ }^{27} \mathrm{Al}$, respectively) of importance for studies of stellar and Galactic chemical evolution. In a Galactic context, $\mathrm{Na}$ is mainly synthesized during hydrostatic carbon burning

\footnotetext{
* Based on observations made with the ESO/VLT, at Paranal Observatory, under program 188.B-3002 (The Gaia-ESO Public Spectroscopic Survey), and on data obtained from the ESO Archive originally observed under programs 60.A-9143, 076.B-0263 and 082.D-0726.

$\star \star$ Table 1 is only available at the CDS via anonymous ftp to cdsarc.u-strasbg.fr (130.79.128.5) or via http://cdsarc.u-strasbg.fr/viz-bin/qcat?]/A+A/589/A115
}

in massive stars (Salpeter 1952; Cameron 1959), where its final abundance is also sensitive to the neutron excess (Woosley $\&$ Weaver 1995). Sodium is also produced in high-temperature H-burning regions through the $\mathrm{NeNa}$ cycle (Salpeter 1955; Denisenkov \& Denisenkova 1990). In low- and intermediatemass stars, Na produced by the NeNa cycle can potentially be mixed to the stellar surface either during the first dredge-up or later during the asymptotic giant branch (AGB) phase (see, e.g., El Eid \& Champagne 1995; Mowlavi 1999; Karakas 2010). Aluminium is mainly synthesized during carbon and neon burning in massive stars (Arnett \& Thielemann 1985; Thielemann \& Arnett 1985). It can also be produced through the $\mathrm{MgAl}$ cycle in the internal convective regions of AGB stars with an initial mass above $\sim 5 M_{\odot}$, which are undergoing hot bottom burning (Ventura et al. 2013; Doherty et al. 2014). 
Abundances of $\mathrm{Na}$ and $\mathrm{Al}$ have been determined in local disk and halo stars in a number of works (e.g., Wallerstein 1962; Spite \& Spite 1980; Peterson 1981; François 1986a,b; Edvardsson et al. 1993; McWilliam et al. 1995; Pilachowski et al. 1996; Carretta et al. 2000; Cayrel et al. 2004; Gehren et al. 2004; Luck \& Heiter 2006; Reddy et al. 2006; Mishenina et al. 2008; Adibekyan et al. 2012; Alexeeva et al. 2014; Bensby et al. 2014). The observed trends with metallicity are different for the two elements. For sodium, a mean trend of increasing $[\mathrm{Na} / \mathrm{Fe}]$ for super-solar metallicities is of particular interest. This is not seen for $[\mathrm{Al} / \mathrm{Fe}]$. The $[\mathrm{Al} / \mathrm{Fe}]$ ratio increases with decreasing metallicity up to $[\mathrm{Al} / \mathrm{Fe}] \sim+0.4$ at $[\mathrm{Fe} / \mathrm{H}] \sim-1.0$, where it decreases again. The increase of $[\mathrm{Na} / \mathrm{Fe}]$ for low metallicities is less pronounced. These trends are discussed in Sect. 4.

Chemical evolution models still have problems reproducing the observed behavior of the $\mathrm{Na}$ and $\mathrm{Al}$ abundances. Depending on the stellar yields adopted by the models, different regions of the $[\mathrm{Na} / \mathrm{Fe}]$ or $[\mathrm{Al} / \mathrm{Fe}]$ vs. $[\mathrm{Fe} / \mathrm{H}]$ diagrams can be fit, but a complete explanation of the detailed trends is not achieved (e.g., Romano et al. 2010; Nomoto et al. 2013). The increase in $[\mathrm{Na} / \mathrm{Fe}]$ for super-solar metallicities is a particular challenge. For example, none of the models computed by Romano et al. (2010), with different stellar yields, was able to reproduce such behavior.

On the stellar evolution side, it is not clear to what extent the first dredge-up (Iben 1964, 1967) in low- and intermediatemass stars is able to bring the products of the NeNa cycle to the stellar photosphere. Stellar evolution models predict that mixing is deep enough to change the $\mathrm{Na}$ abundance only in giants above $\sim 1.5-2.0 M_{\odot}$ (see e.g., Charbonnel \& Lagarde 2010), particularly in those of intermediate-mass above $\sim 4.0 M_{\odot}$ (see e.g., El Eid \& Champagne 1995; Denissenkov 2005). The Al surface abundance is not expected to increase during the giant phase because no magnesium burning is activated in the central region of H-burning of these stars (Weiss \& Charbonnel 2004).

Observationally, it is well known that evolved intermediatemass stars show some $\mathrm{Na}$ enhancement after the first dredgeup (Takeda \& Takada-Hidai 1994; Andrievsky et al. 2002; Kovtyukh et al. 2005; Takeda et al. 2013), although a small excess of $\mathrm{Na}$ from Galactic chemical evolution cannot be fully excluded.

For low-mass stars $\left(0.80 \leq M / M_{\odot} \leq 2.5\right)$, the situation is more confusing. Low-mass metal-poor field giants do not show an indication of changes in their surface $\mathrm{Na}$ abundances (Gratton et al. 2000). However, approximately $65 \%$ of giants in open clusters (stars with higher metallicity and a wider range of masses) seem to have enhanced $\mathrm{Na}$ and/or $\mathrm{Al}$ abundances (see e.g., Jacobson et al. 2007; Smiljanic et al. 2009; Pancino et al. 2010; Carrera \& Pancino 2011; Smiljanic 2012; Yong et al. 2012 , and references therein). Sodium and/or aluminium overabundances are sometimes detected in field giants also (see e.g., Mishenina et al. 2006; Adibekyan et al. 2015).

The level of the Na overabundances varies depending on the study. A combination of different effects seems to cause these disagreements, from the neglect of nonlocal thermodynamical equilibrium (non-LTE) corrections to the use of different atomic data (see e.g., Jacobson et al. 2007; Sestito et al. 2008; Smiljanic 2012; MacLean et al. 2015, and references therein). Therefore, it remains unclear whether there is agreement between stellar evolution models and observed $\mathrm{Na}$ and $\mathrm{Al}$ abundances.

In this work, we take advantage of the Gaia-ESO Survey (Gilmore et al. 2012; Randich \& Gilmore 2013) to study the behavior of $\mathrm{Na}$ and $\mathrm{Al}$ abundances in dwarfs and giants in the context of both stellar and Galactic chemical evolution. This work may be considered a pilot of a larger study to be conducted once the Gaia-ESO survey is completed and many more field stars and open cluster giants are observed. This paper is organized as follows. In Sect. 2 we describe the Gaia-ESO data and their analysis. In Sect. 3 we present a comparison of the observed $\mathrm{Na}$ and $\mathrm{Al}$ abundances in stars of open clusters with stellar evolution models. In Sect. 4 we discuss the comparison of the abundances with Galactic chemical evolution models. Finally, Sect. 5 summarizes our findings.

\section{Data and analysis}

\subsection{Gaia-ESO spectra and analysis}

We use Gaia-ESO Survey ${ }^{1}$ results available in its second and third internal data releases (hereafter iDR2 and iDR3, respectively). Gaia-ESO is a public spectroscopic survey that is conducted with FLAMES (Fiber Large Array Multi-Element Spectrograph, Pasquini et al. 2002) at the European Southern Observatory's (ESO) Very Large Telescope (VLT) in Paranal, Chile.

The Gaia-ESO targets have different spectral types (from O type to $M$ type) and belong to Milky Way fields and to open clusters of different ages and metallicities. Medium- $(R \sim$ $20000)$ and high-resolution $(R \sim 47000)$ spectra are obtained with the Giraffe and UVES (Ultraviolet and Visual Echelle Spectrograph, Dekker et al. 2000) spectrographs, respectively. Here, we use results of the analysis of FGK-type stars observed with UVES (the adopted Giraffe settings do not allow Na measurement). The reduction of these data is described in Sacco et al. (2014).

The analysis details are described in Lanzafame et al. (2015), for stars observed in young open clusters $(\leq 100 \mathrm{Myr})$, and in Smiljanic et al. (2014), for stars observed in the solar neighborhood and open clusters with age $>100$ Myr. Here, we provide only a short description of the procedure; a complete discussion is available in the publications mentioned above.

The spectrum analysis is carried out with multiple pipelines. The two main advantages of this strategy over a single pipeline approach are: 1) one single pipeline is not optimal to analyze stars in all different regions of the parameter space. With multiple pipelines, we can combine their strengths in analyzing, for example, metal-rich and metal-poor stars, dwarfs and giants, or hot and cool stars; and 2) with multiple pipelines, we can investigate the degree to which the different methods agree in each star of the sample, thus quantifying the uncertainties in a way that is not possible with the use of a single pipeline. Such a comparison of multiple pipelines gives an estimate of the precision with which the results can be obtained.

The results of each pipeline were validated using a series of calibrators (Pancino et al., in prep.), which include open and globular cluster stars and the Gaia benchmarks, a set of wellstudied bright stars with fundamental atmospheric parameters (Blanco-Cuaresma et al. 2014; Jofré et al. 2014; Heiter et al. 2015a). For the bulk of our sample stars, analyzed as described in Smiljanic et al. (2014), the final recommended values of atmospheric parameters and abundances are weighted medians of those from the validated methods. Weights are computed with respect to the Gaia benchmarks in a procedure that ties our results to a system of reference defined by atmospheric parameters of these stars. In the Gaia-ESO catalog, each parameter is given together with an estimate of the method-to-method dispersion and the number of pipelines used for its computation.

http://www.gaia-eso.eu 
R. Smiljanic et al.: Stellar and Galactic chemical evolution of $\mathrm{Na}$ and $\mathrm{Al}$

Table 2. Properties of the open clusters for which abundances of $\mathrm{Na}$ and $\mathrm{Al}$ in giants are available.

\begin{tabular}{lccccc}
\hline \hline Name & $\begin{array}{c}\text { Age } \\
(\mathrm{Gyr})\end{array}$ & $\begin{array}{c}{[\mathrm{Fe} / \mathrm{H}]} \\
(\mathrm{dex})\end{array}$ & $\begin{array}{c}M_{\mathrm{TO}} \\
\left(M_{\odot}\right)\end{array}$ & $\begin{array}{c}\mathrm{RV} \\
\left(\mathrm{km} \mathrm{s}^{-1}\right)\end{array}$ & $\begin{array}{c}\# \text { of } \\
\text { giants }\end{array}$ \\
\hline NGC 6705 & 0.316 & $+0.01 \pm 0.06$ & 3.2 & +34.5 & 22 \\
NGC 4815 & 0.630 & $-0.02 \pm 0.04$ & 2.5 & -30.2 & 4 \\
Berkeley 81 & 0.980 & $+0.25 \pm 0.08$ & 2.2 & +47.6 & 13 \\
Trumpler 20 & 1.660 & $+0.09 \pm 0.08$ & 1.8 & -40.5 & 40 \\
NGC 2243 & 3.5 & $-0.44 \pm 0.05$ & 1.2 & +59.5 & 18 \\
Berkeley 25 & 4.5 & $-0.27 \pm 0.02$ & 1.15 & +135.2 & 6 \\
\hline
\end{tabular}

Notes. The mean cluster $[\mathrm{Fe} / \mathrm{H}]$ values are given together with the standard deviation. The cluster $\mathrm{RV}$ is the mean of the giants that we consider to be cluster members. Thus, it can be slightly different from the values adopted to establish membership that are discussed in the text.

\subsection{Sample description}

In the iDR2+iDR3 catalog, atmospheric parameters and abundances are available for 1542 FGK-type stars observed with UVES in the setup with central wavelength $580 \mathrm{~nm}$. To select our sample, first we excluded stars observed in the fields of globular clusters, as their $\mathrm{Na}$ and $\mathrm{Al}$ abundances might be affected by additional processes that would introduce extra complexity in our analysis (see, e.g., Gratton et al. 2012). Second, we restricted the sample to stars with effective temperature $\left(T_{\text {eff }}\right)$ above $4000 \mathrm{~K}$. In the Gaia-ESO releases used here, the results for cooler stars are less reliable because of the increased importance of line blends (for stars analyzed as described in Smiljanic et al. 2014). Future releases are expected to have improvements in this respect. We thus started with a sample of 1303 stars, including 1274 with $\mathrm{Na}$ abundances and 1246 with $\mathrm{Al}$ abundances. All abundances are listed in Table 1. The sample included 957 dwarfs $(\log g>3.50 \mathrm{dex})$ and 346 giants (log $g \leq 3.50 \mathrm{dex}$ ). The sample of solar neighborhood dwarfs (within $\sim 2 \mathrm{kpc}$ of the Sun) includes mostly thin and thick disk objects, and likely few or no halo stars. We do not separate stars of the two disk components, as such a comparison is not one of our goals. The chemical differences between thin and thick disks have been studied with Gaia-ESO Giraffe data by Recio-Blanco et al. (2014), Mikolaitis et al. (2014), and Kordopatis et al. (2015).

The full sample included stars in 16 open clusters. No distinction was made between cluster and field dwarf stars for the Galactic chemical evolution discussion (Sect. 4). For the stellar evolution discussion (Sect. 3), we used giants in NGC 2243, NGC 4815, NGC 6705, Berkeley 25, Berkeley 81, and Trumpler 20 (Table 2). Observations with UVES in these old and intermediate-age open clusters are focused on clump giants. The stars observed in the remaining clusters were all main-sequence or pre-main-sequence stars. We adopted the values of age and turn-off masses obtained using the PARSEC isochrones (Bressan et al. 2012) from earlier Gaia-ESO papers for NGC 6705, NGC 4815, Berkeley 81, and Trumpler 20. For NGC 2243 and Berkeley 25, we derived ages and turn-off masses ourselves, also using PARSEC isochrones for consistency ${ }^{2}$. The metallicities and mean radial velocities in Table 2 are the average of the giants that we considered to be cluster members (see discussion below). These values might be slightly different from the values published in earlier Gaia-ESO papers, which made use of the science verification iDR1. Here we used iDR2 and iDR3, new data releases made after a full reanalysis of the

\footnotetext{
2 For the fitting, we made use of the following photometric data: $V I$ data of Kaluzny et al. (1996) and BVI data of Carraro et al. (2005), for NGC 2243 and Berkeley 25, respectively.
}

whole Gaia-ESO data set that, for some clusters, also included observations of additional stars.

\subsubsection{NGC 6705}

The Gaia-ESO science verification analysis of this cluster was presented in Cantat-Gaudin et al. (2014). We adopt the cluster age derived in that work using PARSEC isochrones, $0.316 \mathrm{Gyr}$, which is similar to other values in the literature, such as the 0.25 Gyr found by Beaver et al. (2013). A total of 49 stars of NGC 6705 were observed. Some of them were AB-type fast rotating main-sequence stars and were thus not considered in our discussion. Abundances of $\mathrm{Na}$ and $\mathrm{Al}$ were available for 24 giants with $T_{\text {eff }}$ above $4000 \mathrm{~K}$. We selected members adopting the mean radial velocity (RV) and dispersion determined by Cantat-Gaudin et al. (2014), i.e., $+34.1 \pm 1.5 \mathrm{~km} \mathrm{~s}^{-1}$, and a three sigma criterium. We found 22 giants to be members.

\subsubsection{NGC 4815}

The Gaia-ESO science verification analysis of NGC 4815 was presented in Friel et al. (2014). Using PARSEC isochrones, they derived an age of $0.63 \mathrm{Gyr}$, which we adopt here. This agrees with the conclusion of Carraro \& Ortolani (1994) that NGC 4815 is about the age of the Hyades. A total of 14 stars were observed in the field of the cluster. For 12 stars with $T_{\text {eff }}>4000 \mathrm{~K}, \mathrm{Na}$ and $\mathrm{Al}$ abundances were available. We selected members using the same RV criterium of Friel et al. (2014), i.e., stars with RV = $-29.4 \pm 4.0 \mathrm{~km} \mathrm{~s}^{-1}$ were considered to be members. Five giants satisfy this criterium, but we only used four of them and further excluded star \# 1795 as it is the most luminous and cool giant of the sample. These characteristics make the analysis of this cool giant more challenging (see discussion in Friel et al. 2014).

\subsubsection{Berkeley 81}

The Gaia-ESO analysis of this cluster was presented in Magrini et al. (2015). The cluster age and turn-off mass found in that analysis (Table 3) are in very good agreement with those found by Donati et al. (2014a), i.e., age $=0.9 \mathrm{Gyr}$ and $M_{\mathrm{TO}}=$ $2.1 M_{\odot}$. Gaia-ESO observations were obtained for 14 giants in the field of Berkeley 81 with UVES. All stars have $T_{\text {eff }}$ above $4000 \mathrm{~K}$ and have available abundances of both $\mathrm{Na}$ and Al. These 14 stars have mean $\mathrm{RV}=+47.5 \pm 0.70 \mathrm{~km} \mathrm{~s}^{-1}$, with a total range between +46.28 and $+48.73 \mathrm{~km} \mathrm{~s}^{-1}$. Hayes \& Friel (2014) performed a RV study of this cluster and found a mean $\mathrm{RV}=+48.1 \pm 2.0$, which is in excellent agreement with the value found here. These authors considered any star with RV within $5 \mathrm{~km} \mathrm{~s}^{-1}$ of the mean value to be member. All of our 14 giants satisfied this criterium, however, the metallicity of one of 
Table 3. Mean abundances of $\mathrm{Na}$ and $\mathrm{Al}$ (with standard deviation) in the giants of each open cluster, after the selection of the best-quality values.

\begin{tabular}{lccccc}
\hline \hline Cluster & $\begin{array}{c}{[\mathrm{Na} / \mathrm{Fe}]} \\
(\mathrm{LTE})\end{array}$ & $\begin{array}{c}{[\mathrm{Na} / \mathrm{Fe}]} \\
(\text { non-LTE) }\end{array}$ & $\begin{array}{c}\text { \# giants with } \\
\text { good Na abun. }\end{array}$ & $\begin{array}{c}{[\mathrm{Al} / \mathrm{Fe}]} \\
(\mathrm{LTE})\end{array}$ & $\begin{array}{c}\text { \# giants with } \\
\text { good Al abun. }\end{array}$ \\
\hline NGC 6705 & $0.42 \pm 0.11$ & $0.38 \pm 0.11$ & 7 & $0.30 \pm 0.04$ & 18 \\
NGC 4815 & $0.17 \pm 0.09$ & $0.13 \pm 0.09$ & 3 & $0.06 \pm 0.05$ & 4 \\
Berkeley 81 & $0.27 \pm 0.06$ & $0.22 \pm 0.06$ & 8 & $0.10 \pm 0.04$ & 12 \\
Trumpler 20 & $0.09 \pm 0.06$ & $0.06 \pm 0.06$ & 31 & $0.02 \pm 0.03$ & 38 \\
NGC 2243 & $0.10 \pm 0.07$ & $0.10 \pm 0.07$ & 15 & $0.07 \pm 0.05$ & 17 \\
Berkeley 25 & 0.05 & 0.04 & 1 & $0.05 \pm 0.01$ & 4 \\
\hline
\end{tabular}

them differs from the mean by more than $3 \sigma$. We considered this star to be a nonmember and excluded it from the stellar evolution discussion.

\subsubsection{Trumpler 20}

The science verification results of Trumpler 20 were published in Donati et al. (2014b). We adopt the age that they derived using PARSEC isochrones, $1.66 \mathrm{Gyr}$, which is similar to the age of 1.5 Gyr obtained by Seleznev et al. (2010). At that time, only 13 giants had been observed and analyzed. We presently have observations for 42 stars. A total of 41 stars have $T_{\text {eff }}$ above $4000 \mathrm{~K}$ and abundances of both $\mathrm{Na}$ and $\mathrm{Al}$. We selected members using the RV criterium of Donati et al. (2014b), i.e., stars with RV within $-40.4 \pm 3.7 \mathrm{~km} \mathrm{~s}^{-1}$ were considered members. A total of 40 giants were retained.

\subsubsection{NGC 2243}

NGC 2243 is one of the most metal-poor open clusters known. A Gaia-ESO analysis of this cluster has not been published yet. The cluster age and turn-off mass that we derived here (Table 3 ) are in very good agreement with those found by Bragaglia \& Tosi (2006), i.e., age $=4.0 \mathrm{Gyr}$ and $M_{\mathrm{TO}}=1.2 M_{\odot}$. We analyzed spectra of 29 different stars: 27 observed by Gaia-ESO and two obtained from the ESO archive. Atmospheric parameters were derived for 26 of them. Based on the RVs, we considered 19 giants to be likely members (mean $\mathrm{RV}=59.5 \pm 0.8 \mathrm{~km} \mathrm{~s}^{-1}$ ). This value is slightly lower than the mean RV of $61.9 \pm 0.8 \mathrm{~km} \mathrm{~s}^{-1}$ found by François et al. (2013) for 82 member stars observed with the Giraffe spectrograph. We further excluded one star with a metallicity higher than that of the others $([\mathrm{Fe} / \mathrm{H}]=-0.17)$, leaving 18 members.

\subsubsection{Berkeley 25}

Ten giants in the field of Berkeley 25 were analyzed. The cluster age that we derived, $4.5 \mathrm{Gyr}$, is in reasonable agreement with the age of 5 Gyr obtained by Carraro et al. (2007). Seven were observed by Gaia-ESO, and three taken from archival data (from the dataset analyzed in Carraro et al. 2007). Abundances were available for nine of them. Two of the stars have an RV that is somewhat discrepant with respect to the others; $\mathrm{RV}=146.5$ and $111.6 \mathrm{~km} \mathrm{~s}^{-1}$ compared to a mean $\mathrm{RV}=135.1 \pm 0.8 \mathrm{~km} \mathrm{~s}^{-1}$ (without the two). A third star seemed to have a somewhat discrepant metallicity $([\mathrm{Fe} / \mathrm{H}]=-0.41)$ when compared to the remaining stars (mean of $-0.23 \pm 0.06$, computed without the discrepant star). This star also has the lowest log $g$ of the sample, therefore increased systematic errors in its parameters cannot be excluded. While we prefer not to draw strong conclusions about membership here, and defer it to a forthcoming publication, for our discussion we considered that only the remaining six giants are members of the cluster.

\subsection{Selecting the best-quality abundances}

A total of up to six $\mathrm{Na}$ lines $(\lambda 4982.814,5153.402,5682.633$, $5688.205,6154.226$, and $6160.747 \AA$ ) and up to three Al lines ( $\lambda$ 5557.063, 6696.023, 6698.673 $\AA$ ) were used to compute the Gaia-ESO abundances. The atomic data were part of the version 4 of the Gaia-ESO line list, details of which will be published elsewhere (see a discussion in Heiter et al. 2015b).

The recommended Gaia-ESO abundances are given in the $\log \epsilon$ format $^{3}$. For each star, a weighted median of the multiple pipeline results, on a line-by-line basis, was computed. The final abundance was then the median of all the line abundances. The median absolute deviation is used as a measurement of the dispersion and can be understood as the precision of the results (see Smiljanic et al. 2014). In Fig. 1 we show the histograms of these dispersions for the $\mathrm{Na}$ and $\mathrm{Al}$ abundances in our sample.

There is an extended tail in the dispersion distribution reaching values above 0.40 dex for both $\mathrm{Na}$ and $\mathrm{Al}$. The third quartile of the dispersion distribution is 0.14 dex for $\mathrm{Na}$ and $0.12 \mathrm{dex}$ for Al. We thus decided to use only abundances with dispersion in $\mathrm{Na}$ or $\mathrm{Al} \leq 0.15$ dex to remove the more uncertain results.

In addition, because of how the recommended parameters and abundances are obtained in Gaia-ESO, the number of pipelines on which the results are based is also important. In Sect. 7.6 of Smiljanic et al. (2014), it is discussed how the accuracy of the recommended atmospheric parameters changes with the use of results from different numbers of pipelines. Similar arguments apply to the accuracy of the abundances.

Results based on fewer pipelines have an increased potential to be more uncertain and thus increase the scatter of the values in the sample. Robust recommended abundances are those based on many determinations, as this guarantees that the distribution of pipeline results (affected by random uncertainties) is well sampled and outlier results are properly identified. In Smiljanic et al. (2014), it was shown that selecting recommended values based on at least five pipelines would guarantee that the majority of the selected results was close to the best possible values.

Here, we decided to use abundances based on determinations from at least four different pipelines. This was a compromise needed to avoid losing too many stars from the sample, which have values coming from only four different pipelines. The effect of stopping at four and not five pipelines will be an increase in the scatter of our abundances. We do not expect the choice to introduce any bias in the results. The abundances in Table 1

\footnotetext{
$3 \log \epsilon(\mathrm{X})=\log [N(\mathrm{X}) / N(\mathrm{H})]+12$, i.e., a logarithmic abundance by number on a scale where the number of hydrogen atoms is $10^{12}$.
} 
R. Smiljanic et al.: Stellar and Galactic chemical evolution of Na and Al
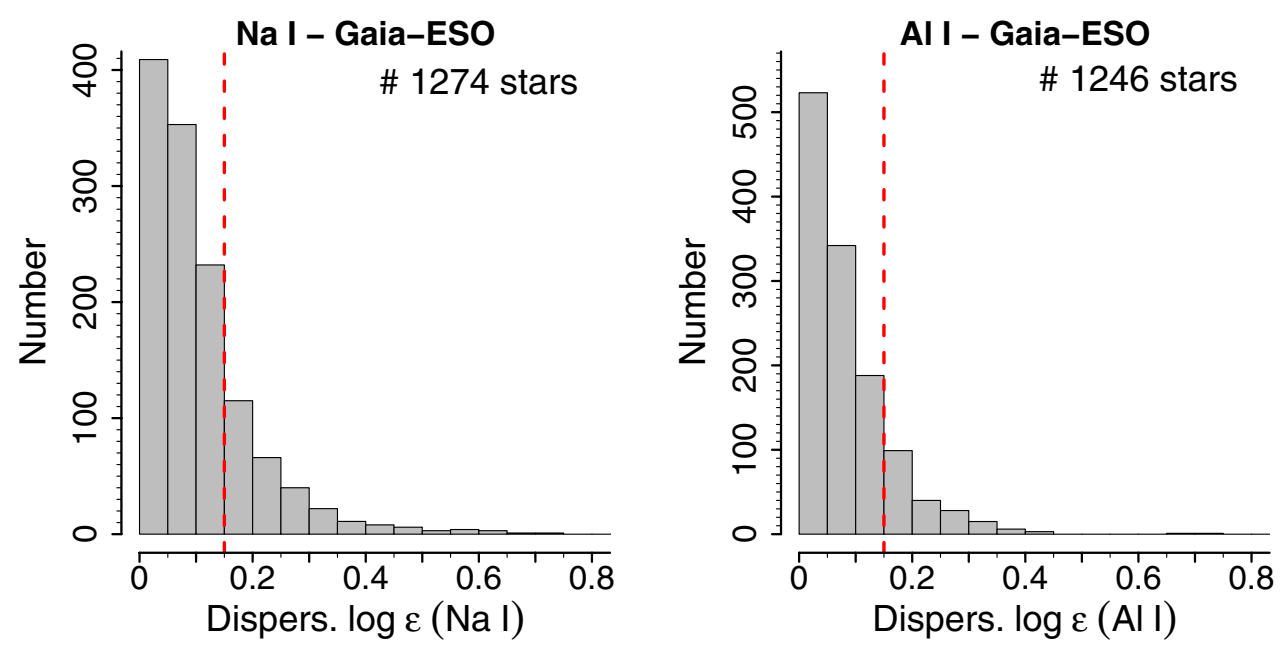

Fig. 1. Precision of the Gaia-ESO Na (left panel) and Al (right panel) abundances available in the iDR2+iDR3 final catalog. The red dashed line indicates the limit of 0.15 dex used to select the best-quality abundances as described in Sect. 2.3.


Fig. 2. Sodium and aluminium abundances as a function of metallicity for the high-quality sample (Sect. 2.3). Dwarfs are shown as blue circles and giants as red squares. Some remaining systematic difference between dwarfs and giants might be present, as discussed in Sect. 2.4 .

are given together with the abundance dispersion and number of pipelines on which they are based.

Of the 13 different pipelines available during the analysis, up to eight provided abundances of $\mathrm{Na}$ and $\mathrm{Al}$, although not for all stars. Internally to Gaia-ESO these eight pipelines are known as Bologna, CAUP, Concepcion, EPINARBO, LUMBA, ParisHeidelberg, UCM, and Vilnius, and are described in Appendix A of Smiljanic et al. (2014).

The restrictions above reduce the sample to 908 stars with $\mathrm{Na}$ abundances ( $\sim 71 \%$ of the original 1274 stars): 237 giants and 631 dwarfs. The sample with $\mathrm{Al}$ abundances is reduced to 941 stars ( $\sim 75 \%$ of the original 1246 stars): 252 giants and 689 dwarfs. These selected abundances are shown as a function of metallicity in Fig. 2. Table 3 lists the mean abundances of $\mathrm{Na}$ and $\mathrm{Al}$, and associated standard deviations, for the selected giants in each open cluster.

\subsection{Systematic effects on the abundances}

The comparison of the abundances in dwarfs and giants, shown in Fig. 2, suggests the possibility of systematic differences. Such differences can appear, for example, because of unidentified line blends that are stronger in a certain type of star.
Weak to moderate trends are indeed present between the LTE $[\mathrm{Na} / \mathrm{Fe}]$ and $[\mathrm{Al} / \mathrm{Fe}]$ ratios with both $T_{\text {eff }}$ and $\log g$. This leads to abundance differences between giants and dwarfs that are clearly seen, for example, in stars belonging to M 67 (Fig. 3). In this cluster, we have a good coverage of the evolutionary track from the main sequence to the red giant branch (RGB). Even though the giants of M 67 are located before the end of the first dredge-up, they show higher $[\mathrm{Na} / \mathrm{Fe}]$ and $[\mathrm{Al} / \mathrm{Fe}]$ ratios in comparison with the dwarfs. The $[\mathrm{Fe} / \mathrm{H}]$ ratio does not show such a trend. This supports that some $\mathrm{Na}$ and $\mathrm{Al}$ differences between dwarfs and giants are likely caused by systematic problems in the analysis.

Because of this, we prefer to be cautious and avoid any discussion comparing the abundances of dwarfs to those of giants. The possibility of systematic differences between dwarfs and giants is being investigated and, if present, will be corrected in new Gaia-ESO data releases.

Within the cluster giants only, there is no correlation between $[\mathrm{Al} / \mathrm{H}]$ and $T_{\text {eff }}$ or $\log g$, either between $[\mathrm{Na} / \mathrm{H}]$ and $\log g$. The metallicity seems to show a weak correlation with $T_{\text {eff }}$ and $\log g$ for stars in Trumpler 20 only. A weak correlation between $[\mathrm{Na} / \mathrm{H}]$ and $T_{\text {eff }}$ is also apparent in Trumpler 20, and suggested by one star in NGC 6705 (but this could eventually be a nonmember outlier). In any case, these weak correlations do not bias 



Fig. 3. Left panel: sample stars from M 67 in the $T_{\text {eff }}-\log g$ plane. Middle panel: $[\mathrm{Na} / \mathrm{Fe}]$ ratio of each star in $\mathrm{M} 67$ as a function of its surface gravity. Right panel: $[\mathrm{Al} / \mathrm{Fe}]$ ratio of each star in $\mathrm{M} 67$ as a function of its surface gravity. Stars are color-coded according to the surface gravity: red for giants with $\log g \leq 3.5$, blue for turn-off and subgiant stars with $3.5<\log g \leq 4.0$, and black for main-sequence stars with $\log g>4.0$. A typical error bar $( \pm 0.14$ dex for [Elem./Fe] and \pm 0.05 dex for $\log g)$ is shown in the upper left part of the middle and right panels.
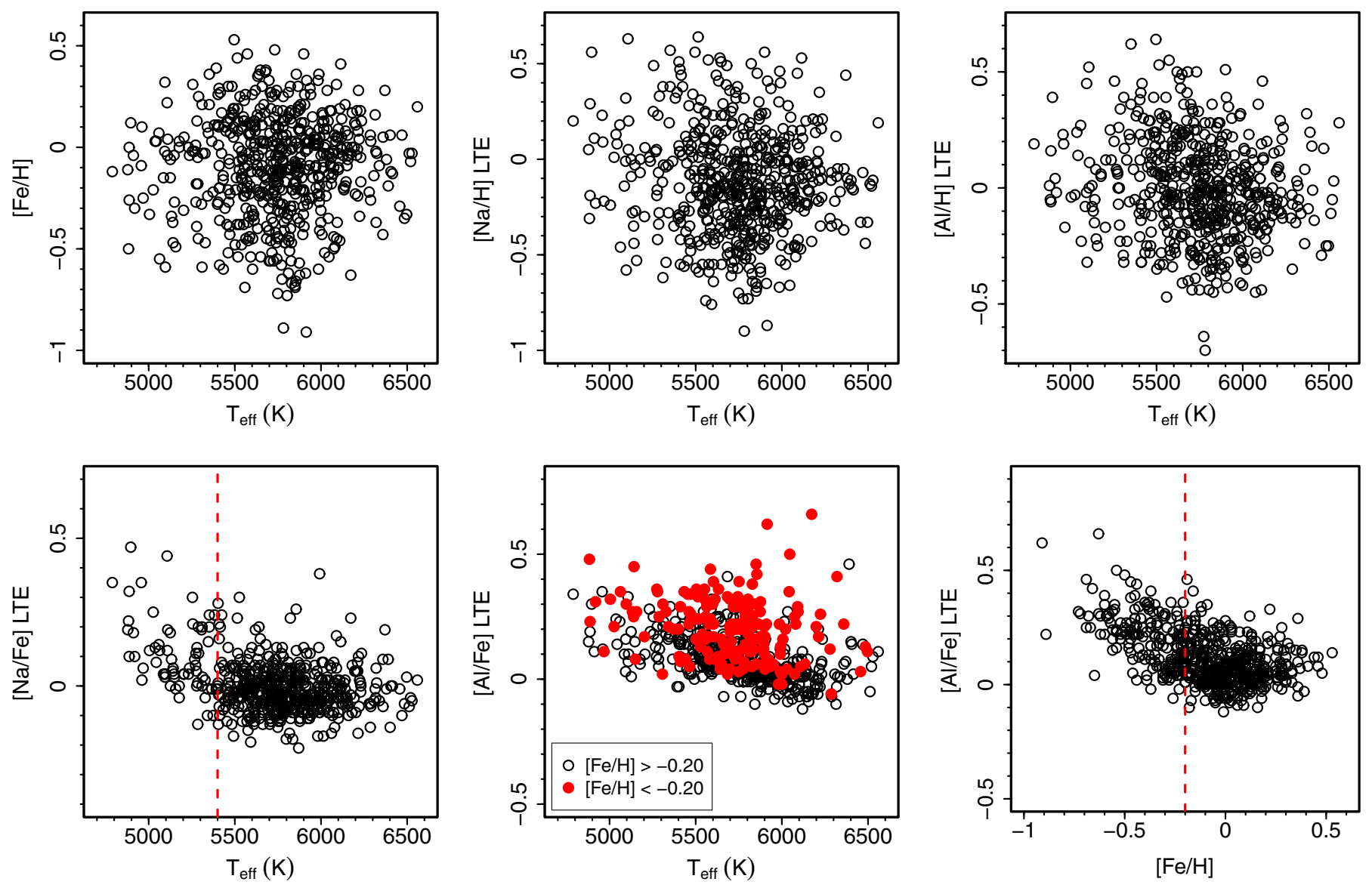

Fig. 4. Trends between abundances and atmospheric parameters in the dwarfs of the sample. Upper left: no apparent trend between [Fe/H] and $T_{\text {eff }}$ (correlation coefficient $\rho=0.03$, with $\mathrm{p}$ value $=0.49)$. Upper middle: weak trend between $[\mathrm{Na} / \mathrm{H}]$ and $T_{\text {eff }}(\rho=-0.09$, with $p$ value $=0.03)$. Upper right: weak trend between $[\mathrm{Al} / \mathrm{H}]$ and $T_{\text {eff }}(\rho=-0.17$, with $p$ value close to zero). Lower left: a moderate trend $(\rho=-0.34$, with $p$ value close to zero) between $[\mathrm{Na} / \mathrm{Fe}]$ and $T_{\text {eff }}$ appears for stars cooler than $5400 \mathrm{~K}$ (red dashed line). Lower middle: a moderate trend between [Al/Fe] and $T_{\text {eff }}(\rho=-0.37$, with $\mathrm{p}$ value close to zero). In red circles, we show the stars with $[\mathrm{Fe} / \mathrm{H}]<-0.20$ selected to understand whether the correlation with $T_{\text {eff }}$ would affect the chemical evolution discussion. Lower right: the trend between $[\mathrm{Al} / \mathrm{Fe}]$ and $[\mathrm{Fe} / \mathrm{H}]$ thought to appear from the Galactic chemical evolution. We selected the stars with $[\mathrm{Fe} / \mathrm{H}]<-0.20$ (left of the red dashed line) to test whether they are the stars mainly affected by the correlation of $[\mathrm{Al} / \mathrm{Fe}]$ with $T_{\text {eff }}$.

the stellar evolution discussion, in which we make use of average abundance ratios per cluster.

Within the dwarfs, there is no trend of $[\mathrm{Fe} / \mathrm{H}]$ with $T_{\text {eff }}$, but weak trends are suggested between $[\mathrm{Fe} / \mathrm{H}]$ and $\log g$, and between $[\mathrm{Na} / \mathrm{H}]$ and $[\mathrm{Al} / \mathrm{H}]$ and both $T_{\text {eff }}$ and $\log g$ (top row of
Fig. 4). These weak trends are mostly imperceptible by eye, as there is a large scatter at each value of the atmospheric parameters. For $[\mathrm{Al} / \mathrm{Fe}]$ and $[\mathrm{Na} / \mathrm{Fe}]$, no trend is apparent with $\log g$. However, moderate trends appear when looking at both $[\mathrm{Na} / \mathrm{Fe}]$ and $[\mathrm{Al} / \mathrm{Fe}]$ as a function of $T_{\text {eff }}$ (bottom row of Fig. 4). 
R. Smiljanic et al.: Stellar and Galactic chemical evolution of $\mathrm{Na}$ and $\mathrm{Al}$

Table 4. Results of multiple analyses of solar spectra in the Gaia-ESO Survey for the iDR2 and iDR3 cycles.

\begin{tabular}{lccccccc}
\hline \hline Spectrograph & Cycle & $\begin{array}{c}T_{\text {eff }} \\
(\mathrm{K})\end{array}$ & $\begin{array}{c}\log g \\
(\mathrm{dex})\end{array}$ & $\begin{array}{c}{[\mathrm{Fe} / \mathrm{H}]} \\
(\mathrm{dex})\end{array}$ & $\begin{array}{c}\xi \\
\left(\mathrm{km} \mathrm{s}^{-1}\right)\end{array}$ & $\begin{array}{c}\log \epsilon(\mathrm{Na}) \\
(\mathrm{dex})\end{array}$ & $\begin{array}{c}\log \epsilon(\mathrm{Al}) \\
(\mathrm{dex})\end{array}$ \\
\hline FLAMES/UVES & iDR2 & $5826 \pm 40$ & $4.50 \pm 0.05$ & $-0.03 \pm 0.12$ & $1.05 \pm 0.00$ & $6.31 \pm 0.05$ & $6.44 \pm 0.01$ \\
FLAMES/UVES & iDR3 & $5797 \pm 85$ & $4.45 \pm 0.11$ & $0.03 \pm 0.03$ & $0.70 \pm 0.25$ & $6.27 \pm 0.07$ & $6.43 \pm 0.09$ \\
NARVAL & iDR2 & $5810 \pm 17$ & $4.50 \pm 0.08$ & $0.00 \pm 0.04$ & $1.06 \pm 0.08$ & $6.29 \pm 0.02$ & $6.46 \pm 0.01$ \\
NARVAL & iDR3 & $5785 \pm 40$ & $4.44 \pm 0.14$ & $0.03 \pm 0.12$ & $0.94 \pm 0.20$ & $6.30 \pm 0.13$ & $6.43 \pm 0.04$ \\
UVES stand-alone & iDR2 & $5777 \pm 31$ & $4.43 \pm 0.13$ & $0.00 \pm 0.06$ & $1.04 \pm 0.16$ & $6.28 \pm 0.02$ & $6.45 \pm 0.03$ \\
UVES stand-alone & iDR3 & $5774 \pm 25$ & $4.43 \pm 0.10$ & $0.04 \pm 0.04$ & $0.95 \pm 0.17$ & $6.33 \pm 0.07$ & $6.44 \pm 0.05$ \\
\hline Average & - & $5795 \pm 20$ & $4.46 \pm 0.03$ & $0.01 \pm 0.03$ & $0.96 \pm 0.14$ & $6.30 \pm 0.02$ & $6.44 \pm 0.01$ \\
\hline
\end{tabular}

Notes. The metallicities, [Fe/H], are given with respect to $\log \epsilon(\mathrm{Fe})=7.45$ from Grevesse et al. (2007).

For $\mathrm{Na}$, the only effect of the stars with $T_{\text {eff }}<5400 \mathrm{~K}$ in the $[\mathrm{Na} / \mathrm{Fe}]$ vs. $[\mathrm{Fe} / \mathrm{H}]$ plot is to increase the scatter. No systematic bias is introduced. Nevertheless, we decided to exclude such cool stars from the discussion of the chemical evolution of $\mathrm{Na}$, as it is simple enough to include a temperature cut in the sample. For $\mathrm{Al}$, however, the trend of $[\mathrm{Al} / \mathrm{Fe}]$ with $T_{\text {eff }}$ is not restricted to a given temperature range. However, as can be seen in the bottom row of Fig. 4, the rise of $[\mathrm{Al} / \mathrm{Fe}]$ at low metallicities is not caused by the systematic trend with $T_{\text {eff }}$. It seems again that this trend with temperature only affects the scatter of the points, and does not introduce further systematic effects in the interpretation of the chemical evolution of Al. We therefore do not include additional restrictions in the sample of dwarfs with $\mathrm{Al}$ abundances.

\subsection{The solar abundances}

As solar reference abundances we used the results obtained from the analysis of the FLAMES/UVES solar spectrum ${ }^{4}$ in GaiaESO iDR2, as presented in Smiljanic et al. (2014), and listed in the first line of Table 4.

However, Gaia-ESO also analyzed a solar NARVAL ${ }^{5}$ spectrum and the UVES (obtained in stand-alone mode) spectrum from the Gaia benchmark stars library (Blanco-Cuaresma et al. 2014). The three spectra were analyzed once during iDR2 and again for iDR3. With these multiple analyses (Table 4), we were able to investigate the uncertainties on our solar reference abundances.

This comparison reveals a variation of up to $0.07 \mathrm{dex}$ in [Fe/H], of up to 0.06 dex in $\log \epsilon(\mathrm{Na})$, and of up to 0.03 dex in $\log \epsilon(\mathrm{Al})$. These differences reflect the use of different pipelines to define the recommended parameters of the Sun in each GaiaESO internal release, which is an effect similar to what was discussed in Sect. 2.3. We remark that the Sun was analyzed as any other star in our sample. The Sun is used as one of the benchmark stars, but no special weight is given to its analysis with respect to the other benchmarks. Thus, by itself the Sun does not define our system of parameters and abundances, but is one of the stars defining that system. The differences in the solar parameters and abundances as listed in Table 4 do not reflect changes in the scales as much as they quantify uncertainties inherent in our method of defining the recommended results. Nevertheless, a solar analysis can be used as a special reference when we need to list abundances in the [Element/Fe] format. We prefer this approach, over adopting reference solar abundances from literature compilations, as our own solar analyses reflect better shortcomings such as lack of non-LTE corrections.

\footnotetext{
4 http://www.eso.org/observing/dfo/quality/GIRAFFE/ pipeline/solar.html

5 NARVAL is a spectropolarimeter on the $2 \mathrm{~m}$ Telescope Bernard Lyot (TBL) atop Pic du Midi (Aurière 2003).
}

The important observation from Table 4 is that most values (parameters and abundances) agree with each other, within their uncertainties. However, the variation in the solar abundances has an impact on the abundance ratios of the sample. Depending on which solar analysis is used as reference, there can be a maximum change of up to $0.10 \mathrm{dex}$ in $[\mathrm{Na} / \mathrm{Fe}]$ and up to $0.07 \mathrm{dex}$ in $[\mathrm{Al} / \mathrm{Fe}]$. This is an intrinsic uncertainty of the zero point of our abundance scale and is important when comparing the element ratios to the predictions of the stellar evolution models. Nevertheless, this has no influence on the relative comparison between stars of the sample, as a zero point change would affect all stars in the same way. Moreover, we normalize the chemical evolution model predictions to our adopted solar abundances, therefore the comparison with the observations in this case is also not affected.

\subsection{Non-LTE corrections}

The Na abundances were corrected for non-LTE effects using the grids of Lind et al. (2011). The corrections were derived on a line-by-line basis, using the atmospheric parameters and LTE Na abundance of each star as input. Two of the lines used in GaiaESO, $\lambda 4982.814$ and 5153.402 $\AA$, were not part of the original grid. Nevertheless, they were part of the model atom of that work and thus the corrections for them could be computed.

The average corrections for all stars (giants and dwarfs) are always negative and range from -0.06 down to -0.18 dex. For a few stars, the non-LTE correction was actually extrapolated. This was the case for stars with $\xi<1.00 \mathrm{~km} \mathrm{~s}^{-1}$ (119 stars) and for two stars with $[\mathrm{Fe} / \mathrm{H}]>+0.50 \mathrm{dex}$, as these values are also outside the original grid. For a few other stars (24), it was not possible to compute non-LTE corrections because their LTE $\mathrm{Na}$ abundances had values outside the Lind et al. (2011) grid. For our reference Sun, the non-LTE correction is of $-0.08 \mathrm{dex}$ and the non-LTE abundance of $\mathrm{Na}$ is thus $\log \epsilon(\mathrm{Na})=6.23$. In Table 1, we give the non-LTE $[\mathrm{Na} / \mathrm{Fe}]$ ratio for those stars with good quality abundances (as described in Sect. 2.3).

We did not correct the $\mathrm{Al}$ abundances for non-LTE effects. Although non-LTE abundances of Al have been computed in the literature (e.g., Baumueller \& Gehren 1997; Gehren et al. 2004; Andrievsky et al. 2008), no comprehensive grid of corrections is currently available for the metallicity range of our sample.

Instead, we estimated non-LTE corrections for $\mathrm{Al}$ from new preliminary computations performed by one of us (T. Nordlander). The calculations were carried out for $\lambda 5557$ and 6696-6698 $\AA$ lines and for stellar parameters and abundances representative of the giants in the open clusters, for the Sun and for two additional sets of dwarf-like parameters. The non-LTE model, which will be described in an upcoming paper (Nordlander et al., in prep.), adopts realistic hydrogen collisional 
rates (Belyaev 2013; Kaulakys 1991) as well as newer electron collisional rates.

The average corrections for the giants seem to be approximately -0.05 dex. We are not aware of other published non-LTE corrections for $\mathrm{Al}$ in solar-metallicity giants, and thus cannot compare with previous results. For the Sun and the two dwarfs, the average corrections are small, about $-0.01 /-0.02$ dex. This agrees with the results of Baumueller \& Gehren (1996), who found that abundances derived from the lines at 6696 and $6698 \AA$ agree in LTE and non-LTE to within 0.01 dex in the Sun. The different corrections for dwarfs and giants are not sufficient to explain the difference in $[\mathrm{Al} / \mathrm{Fe}]$ between the types of stars that were discussed in Sect. 2.4.

\subsection{Ages for field dwarfs}

Ages and masses were computed for field dwarf stars following the procedure described in Bergemann et al. (2014). This is accomplished with the Bellaterra Stellar Parameter Pipeline (Serenelli et al. 2013), which adopts a grid of stellar evolutionary tracks computed with the GARSTEC code (GARching STellar Evolution Code, Weiss \& Schlattl 2008). As in Bergemann et al. (2014), we only use ages and masses obtained for stars with $\log g>3.5$, as the models are degenerate outside this regime, and for which the fractional age error is $<30 \%$. The age accuracy is of course limited by the accuracy of the atmospheric parameters used in its computation and by the accuracy of the stellar models. The age values are available for 381 dwarfs in our sample. We note the use of different stellar models to compute the ages of open clusters and field stars, which likely results in two different scales. However, these two sets of ages are not discussed together. Moreover, the errors in age among the field stars are likely larger than any systematic between the two stellar models.

\section{Stellar evolution with open cluster stars}

There is some discussion in the literature about overabundances of $\mathrm{Na}$ and $\mathrm{Al}$ in giants of open clusters and a possible connection with the first dredge-up (see, e.g., Jacobson et al. 2007; Smiljanic 2012, and references therein). In this section, we revisit this issue using the Gaia-ESO sample of open cluster giants described above. The vast majority of the giants observed by Gaia-ESO are expected to be clump giants, and thus after completion of the first dredge-up.

We complemented the Gaia-ESO results with the Na abundance of clump giants in the Hyades open cluster determined by Smiljanic (2012). The Na overabundance in the Hyades giants (age of $\sim 625 \mathrm{Myr}$ and turn-off mass $\sim 2.58 M_{\odot}$ ) was first found by Helfer \& Wallerstein (1964). With well constrained atmospheric parameters (mostly independent of spectroscopy), and a critical selection of spectral lines, Smiljanic (2012) found $[\mathrm{Na} / \mathrm{Fe}]=+0.30$ in non-LTE (also corrected using the grid of Lind et al. 2011). The adopted $g f^{6}$ values of the $\mathrm{Na}$ lines were the same as those used here, but accounting for differences in the solar reference abundances (but not in the stellar parameters scale), the Hyades have $[\mathrm{Na} / \mathrm{Fe}]=+0.26$ in the Gaia-ESO scale.

To compare with the observations, we use the evolutionary models computed by Lagarde et al. (2012) and Ventura et al. (2013). The models of Ventura et al. (2013) include only

\footnotetext{
6 The product of the statistical weight $g$ of the lower energy level involved in the transition with the transition oscillator strength $f$.
}

convection as a mixing mechanism and were computed for two metallicities, solar and $[\mathrm{Fe} / \mathrm{H}]=-0.40$. The Lagarde et al. (2012) models were computed for solar metallicity and $[\mathrm{Fe} / \mathrm{H}]=-0.54$, and for the cases with and without rotation-induced mixing. The model with rotation also includes thermohaline mixing, but this process does not affect $\mathrm{Na}$ or $\mathrm{Al}$. The initial rotation velocity of the modeled stars is $30 \%$ of the critical velocity at the zero-age main sequence (see Lagarde et al. 2014). If the initial rotation of the observed stars was different from that, the effect of rotation induced-mixing would also be different. Therefore, some scatter at a given mass can be expected, reflecting the scatter in the initial rotation of stars of the same mass. However, we do not have models computed with different initial rotation values and cannot judge the expected magnitude of such scatter.

We do not renormalize the models to our adopted solar abundances. We consider both the observed and modeled $[\mathrm{Na} / \mathrm{Fe}]$ and $[\mathrm{Al} / \mathrm{Fe}]$ values to be relative values with respect to the abundances that the stars had during the main sequence. While this is strictly true for the models, as the stars had initially $[\mathrm{Na} / \mathrm{Fe}]$ and $[\mathrm{Al} / \mathrm{Fe}]=0.0$, for the observed giants the main-sequence $\mathrm{Na}$ and $\mathrm{Al}$ abundances are unknown. For the effects of this discussion, we assume that the Sun is a good reference for the initial $\mathrm{Na}$ and $\mathrm{Al}$ abundances of the stars, which by definition implies $[\mathrm{Na} / \mathrm{Fe}]$ and $[\mathrm{Al} / \mathrm{Fe}]=0.0$. An expanded discussion of dwarfs and giants abundances in a few open clusters will be possible with new Gaia-ESO observations, and will be the subject of a future paper.

\subsection{Model comparison with the Gaia-ESO sodium abundances}

As can be seen in the left panel of Fig. 5, according to the stellar evolution models stars less massive than $\sim 1.5-2.0 M_{\odot}$ do not change their Na surface abundance after the first dredge-up. For more massive stars, a change in the surface $\mathrm{Na}$ abundance is expected. In the Lagarde et al. (2012) models, the higher the stellar mass, the stronger the overabundance (for both models, with and without rotation). In the Ventura et al. (2013) models, instead, the expected $\mathrm{Na}$ enhancement is constant above $\sim 3.0 \mathrm{M}_{\odot}$.

Regarding the observations, our results indicate that even when non-LTE effects are taken into account, some giants in open clusters still display $\mathrm{Na}$ overabundances with respect to the Sun. The observations also suggest an increase in $\mathrm{Na}$ enhancement as a function of stellar mass. However, with only one cluster beyond $\sim 3.0 M_{\odot}$, we cannot state whether the Na overabundance continues to increase or reaches a plateau.

Because of the zero point uncertainty in $[\mathrm{Na} / \mathrm{Fe}]$, we cannot exclude a small $\mathrm{Na}$ overabundance below $\sim 2 M_{\odot}$. One cluster in particular, NGC 2243 with turn-off mass $\sim 1.2 M_{\odot}$, displays a mild overabundance, $[\mathrm{Na} / \mathrm{Fe}]=+0.10 \pm 0.07$ (average and standard deviation), although still in marginal agreement with the models within the errors. This is the most metal-poor cluster in our sample, $[\mathrm{Fe} / \mathrm{H}]=-0.44$. Nevertheless, the low-metallicity models for this mass range behave the same as the solar metallicity models, i.e., no $\mathrm{Na}$ overabundance is expected.

For stars above $\sim 2 M_{\odot}$ we can draw stronger conclusions. The $\mathrm{Na}$ overabundances are real, are not erased when non-LTE is taken into account, and they seem to increase with increasing stellar mass. The zero point uncertainty does not change this conclusion. As the Sun is a reference for all $[\mathrm{Na} / \mathrm{Fe}]$ abundances, the values could move up or down in Fig. 5, but the overabundances would not disappear completely and the trend with stellar mass would remain.

In addition, the Na overabundances would likely remain in an analysis using more realistic three-dimensional (3D) model 



Fig. 5. Mean cluster abundance, from giants only, after the selection of members and best-quality values. We estimate the uncertainty in the turn-off masses to be less than $\pm 0.1 M_{\odot}$.

atmospheres. Collet et al. (2007) and Dobrovolskas et al. (2013) have compared $\mathrm{Na}$ abundances of giants derived using 1D and $3 \mathrm{D}$ model atmospheres, for a few representative cases. For the Na lines 6154 and $6160 \AA$, with excitation potential $\sim 2 \mathrm{eV}$, the corrections are small $(\leq \pm 0.05 \mathrm{dex})$ and could be positive (i.e., the 3D corrected $\mathrm{Na}$ abundances could be slightly larger than our values based on a 1D analysis).

Thus, we consider the trend in Fig. 5 real and a strong indication that the sodium overabundances in these stars are caused by internal evolutionary processes. In the future, new Gaia-ESO observations of giants in young clusters (age $~ 100$ Myr; e.g., NGC 3532, NGC 6067, and NGC 6633) will help to further populate the high-mass end of Fig. 5. This will help to expand the current discussion, and perhaps provide an opportunity to discriminate between models with and without rotation at the high-mass end. We note that, as reported in Tautvaišienè et al. (2015), the $\mathrm{C}$ and $\mathrm{N}$ abundances in clump giants of NGC 4815 and NGC 6705, and in both clump and evolved RGB stars in Trumpler 20 seem to agree better with models without rotation, although the models with rotation cannot be excluded because of their large error bars.

\subsection{Model comparison with the Gaia-ESO aluminium abundances}

The right panel of Fig. 5 suggests that below $3 M_{\odot}$ the LTE abundance of $\mathrm{Al}$ in giants is constant around $[\mathrm{Al} / \mathrm{Fe}] \sim+0.06$. Taking an average non-LTE correction into account on the order of -0.05 dex (Sect. 2.6), we find that the stars below $3 M_{\odot}$ are consistent with $[\mathrm{Al} / \mathrm{Fe}]=0.00$, i.e., no change in the surface abundance of $\mathrm{Al}$ after the first dredge-up. Thus, the observations agree well with the predictions of stellar evolution models. The small scatter in the observed abundances is consistent with the uncertainties. Even though there is some uncertainty in the zero point of our $[\mathrm{Al} / \mathrm{Fe}]$ values (a maximum change of $0.07 \mathrm{dex}$ ), the lack of trend with stellar mass is a good indicator that there is no stellar evolutionary effect in the $\mathrm{Al}$ abundances.

The only cluster above $3 M_{\odot}$, NGC 6705 , seems to have an enhanced $\mathrm{Al}$ abundance $([\mathrm{Al} / \mathrm{Fe}]=+0.30 \mathrm{dex}$ in LTE), which would remain significant even after non-LTE corrections. However, we remark that stars in NGC 6705 seem to be $\alpha$-enhanced (as discussed in Magrini et al. 2014, 2015). While $\mathrm{Al}$ is not an $\alpha$-element, it does seem to behave as one, at least for metallicities between solar and $[\mathrm{Fe} / \mathrm{H}] \sim-1.0$ (see Fig. 2). We cannot discard the possibility that the $\alpha$-enhancement of NGC 6705 is accompanied by a similar Al enhancement. In fact, we note that the disk field stars analyzed by Bensby et al. (2014) that have $[\mathrm{Fe} / \mathrm{H}]>0.00$ and $[\mathrm{Mg} / \mathrm{Fe}]>+0.1$ are also enhanced in Al. Thus, the Al overabundance in NGC 6705 seems to be related to the environment where the cluster was formed. Indeed, Magrini et al. (2015) made the hypothesis that NGC 6705 was enriched by a type II supernova in the mass range $15-18 M_{\odot}$. The measurement of $\mathrm{Al}$ abundances in dwarfs of this cluster would help to clarify the situation, but in our sample $\mathrm{Al}$ abundances are only available for giants. New Gaia-ESO observations of giants in young clusters will also be useful in this context.

\subsection{Na enhancement: Literature results}

\subsubsection{Open clusters}

We now check whether literature $\mathrm{Na}$ abundances support our conclusions above. For this, we take advantage of the compilation of $\mathrm{Na}$ abundances by MacLean et al. (2015). These authors conducted a homogenization of literature $\mathrm{Na}$ abundances in open cluster stars, changing the solar reference abundances and applying the non-LTE corrections of Lind et al. (2011).

We extracted the $\mathrm{Na}$ abundances obtained only from the analysis of giants from their Table 2. This included a total of eleven open clusters, but we further excluded NGC 6791. For this cluster, the compilation listed the $\mathrm{Na}$ abundances from Geisler et al. (2012). These authors claimed to observe a Na-O anticorrelation similar to the anticorrelation common in globular clusters. We do not include these results to avoid introducing a different physical effect in the discussion. We also remark that the Na-O anticorrelation in NGC 6791 was not confirmed by both Bragaglia et al. (2014) and Cunha et al. (2015), and that Boesgaard et al. (2015) did not find any spread of oxygen abundances in turn-off stars of the cluster.

Figure 6 shows the $[\mathrm{Na} / \mathrm{Fe}]$ ratios extracted from MacLean et al. (2015) as a function of the turn-off mass of the clusters. Ages and turn-off masses for the ten clusters (i.e., Berkeley 39, Collinder 261, Hyades, IC 4651, M 67, NGC 3114, NGC 6134, 


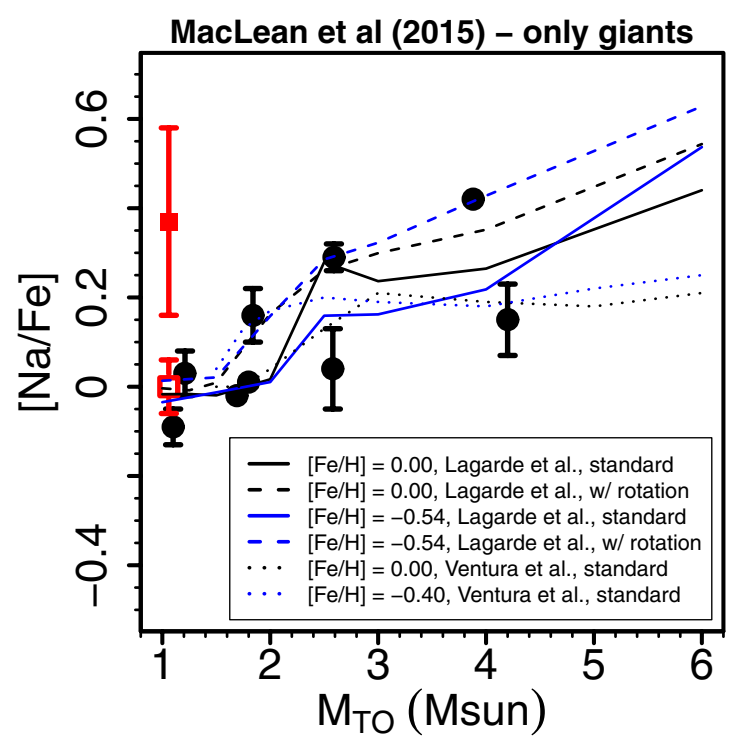

Fig. 6. Mean $[\mathrm{Na} / \mathrm{Fe}]$, in non-LTE, only of giants for clusters in the compilation of MacLean et al. (2015). The red solid square is the original $[\mathrm{Na} / \mathrm{Fe}]$ value of Collinder 261 in that compilation, while the red open square is our revised value as discussed in the text.

NGC 6475, NGC 7789, and Trumpler 20) were taken from a variety of references. These include some values of turn-off masses that we adopted in this work (e.g., for the Hyades and Trumpler 20) and those quoted in the original sources of the abundances (Tautvaišienè et al. 2000, 2005; Friel et al. 2003; Carretta et al. 2005; Schuler et al. 2009; Villanova et al. 2009; Mikolaitis et al. 2010, 2011; Bragaglia et al. 2012; Santrich et al. 2013; Carraro et al. 2014). Apart from one cluster, Collinder 261 at $1.06 M_{\odot}$ and $[\mathrm{Na} / \mathrm{Fe}]=+0.37$, Fig. 6 shows a trend of $[\mathrm{Na} / \mathrm{Fe}]$ with stellar mass similar to that seen in our own sample. The scatter seems to be larger, but we note that this is a compilation of literature results. In addition, we do not know for certain the evolutionary status of all these giants. Some might be before the end of the first dredge-up. Thus we refrain from over interpreting the scatter and postpone a more detailed discussion for when a larger sample of homogeneous Gaia-ESO results become available.

The abundances of Collinder 261 are originally from Friel et al. (2003), $[\mathrm{Fe} / \mathrm{H}]=-0.22$ and $[\mathrm{Na} / \mathrm{Fe}]=+0.48 \pm 0.22$ (in LTE); and Carretta et al. (2005), $[\mathrm{Fe} / \mathrm{H}]=-0.03$ and $[\mathrm{Na} / \mathrm{Fe}]=+0.33 \pm 0.06$ (in non-LTE, with corrections from Gratton et al. 1999). Correcting these values for the MacLean et al. (2015) solar scale, we obtain $[\mathrm{Na} / \mathrm{Fe}]=+0.55$ and $[\mathrm{Na} / \mathrm{Fe}]=+0.26$, respectively. Carretta et al. discussed the difference between the two results listing as possible reasons, for example, the higher spectral resolution of their own data and their more robust determination of microturbulence. We thus prefer to adopt the Carretta et al. analysis as the reference $\mathrm{Na}$ abundance for Collinder 261.

The Na non-LTE correction of Gratton et al. (1999) for a star of $T_{\text {eff }}=4000 \mathrm{~K}$ and $\log g=1.5 \mathrm{dex}$ is on the order of $+0.20 \mathrm{dex}^{7}$. As discussed before, the average correction from the Lind et al. (2011) grid, however, is on the order of -0.10 dex. Taking this difference into account, the non-LTE Na abundance for Collinder 261 would instead be $[\mathrm{Na} / \mathrm{Fe}] \sim 0.00$.

\footnotetext{
Online table available at Vizier: http://vizier.cfa.harvard. edu/viz-bin/VizieR-3?- source=]/A\%2bA/350/955/abundcor
}

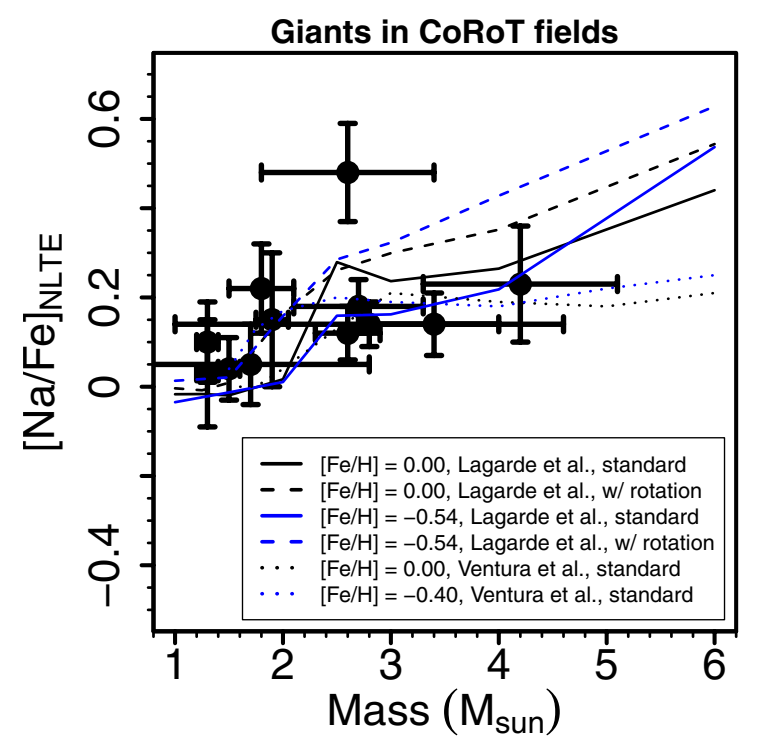

Fig. 7. Sodium abundances as a function of stellar mass for a sample of literature giants with seismic data.

With this revised value, the $\mathrm{Na}$ abundance in Collinder 261 turns out to be in excellent agreement with the model expectations for its turn-off mass. These literature results support the trend of increasing $\mathrm{Na}$ overabundance with increasing stellar mass and, moreover, also suggest that there is no significant $\mathrm{Na}$ overabundance for low-mass stars below 2.0 $M_{\odot}$. This supports the idea that evolutionary mixing processes are the origin of the observed $\mathrm{Na}$ enhancements in giants with $M \gtrsim 2.0 M_{\odot}$.

\subsubsection{Giants with seismic masses}

It is not straightforward to look for the $[\mathrm{Na} / \mathrm{Fe}]$ vs. stellar mass trend in field giants because accurate masses and evolutionary stages are notoriously difficult to determine for field stars. This has started to change with the advent of asteroseismic space missions, such as CoRoT (Convection, Rotation, and planetary Transits, Baglin et al. 2006) and Kepler (Borucki et al. 2010). By studying the oscillation properties of giants it has become possible to estimate their masses, among other quantities (see e.g., Stello et al. 2008; Kallinger et al. 2010; Mosser et al. 2010).

Taking advantage of this, we extracted a sample of 16 giants with LTE Na abundances from Morel et al. (2014), which are determined using a set of atmospheric parameters including $\log g$ based on the ionization equilibrium. Seismic masses for the same giants were adopted from Lagarde et al. (2015). The giants have metallicities between $[\mathrm{Fe} / \mathrm{H}]=-0.35$ and +0.13 . Twelve of these giants have been observed by CoRoT, while the other four are bright well-studied giants with asteroseismic data available from elsewhere and used as reference stars by Morel et al. (2014). We computed non-LTE Na abundance corrections again using the grids computed by Lind et al. (2011).

The $[\mathrm{Na} / \mathrm{Fe}]$ ratios of these giants are shown as a function of stellar mass in Fig. 7. This data set corroborates our previous conclusions. A similar trend between $[\mathrm{Na} / \mathrm{Fe}]$ and stellar mass is seen here. Stars more massive than $\sim 2.0-2.5 M_{\odot}$ have on average higher $\mathrm{Na}$ enhancement than stars less massive than that. The one outlier with higher $[\mathrm{Na} / \mathrm{Fe}]$ than expected by the models is HD 50890. This star, however, has unusually large line broadening (Morel et al. 2014) and, according to the more detailed analysis of Baudin et al. (2012) using the same CoRoT data, could have a mass of up to $5 M_{\odot}$. The higher mass would improve the agreement with the models. 

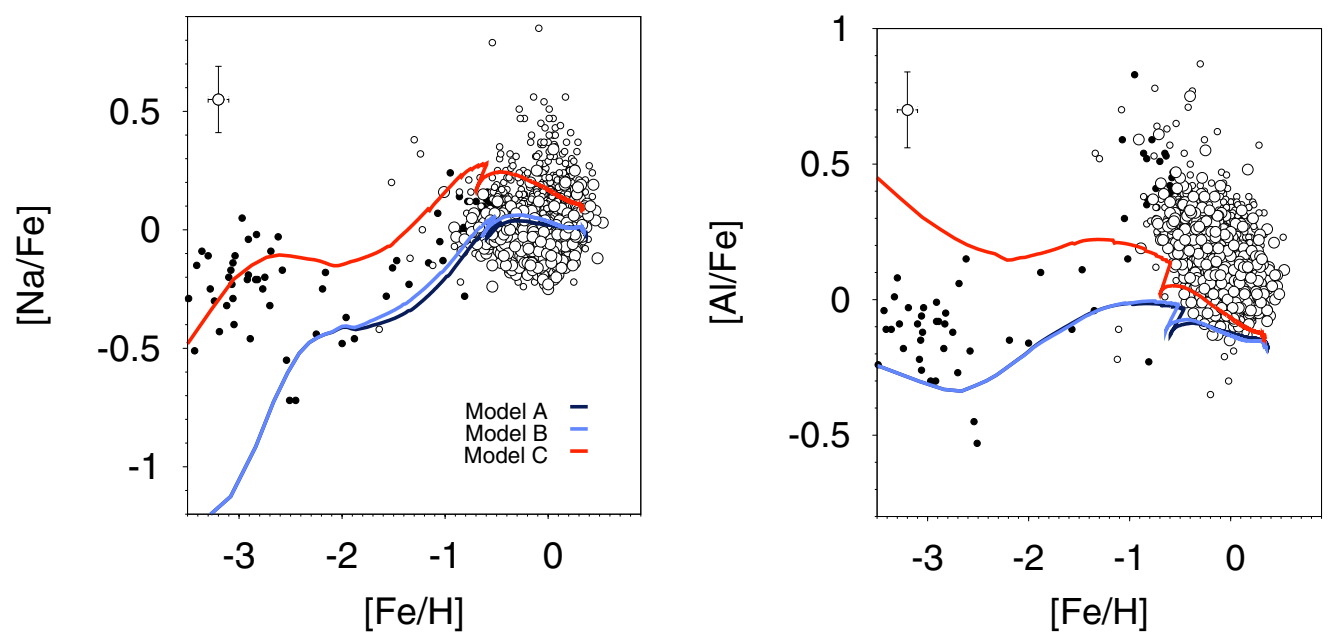

Fig. 8. Runs of $[\mathrm{Na} / \mathrm{Fe}]$ (left panel) and $[\mathrm{Al} / \mathrm{Fe}]$ (right panel) with $[\mathrm{Fe} / \mathrm{H}]$ predicted by chemical evolution models for the solar neighborhood adopting different stellar yields (see text; legend on the lower right corner of the left panel). Data for low-metallicity stars (filled circles) are from Gehren et al. (2006), Andrievsky et al. (2007, for Na only), and Andrievsky et al. (2008, for Al only). High-quality data for our sample dwarfs (selected as in Sect. 2.3) are shown as large empty circles, while the small empty circles refer to the full sample, including lower quality data and giants. All measured $\mathrm{Na}$ abundances were corrected for non-LTE effects. Typical error bars are $\sim 0.15 \mathrm{dex}$ for $[\mathrm{El} / \mathrm{Fe}]$ and $\sim 0.10 \mathrm{dex}$ for $[\mathrm{Fe} / \mathrm{H}]$ in this and all following plots. A typical error bar $( \pm 0.10$ dex for $[$ Elem./H] and \pm 0.14 dex for [Elem./Fe] is shown in the upper left of each panel. $)$

\section{Galactic chemical evolution of $\mathrm{Na}$ and $\mathrm{Al}$}

The history of $\mathrm{Na}$ and $\mathrm{Al}$ enrichment on a Galactic scale is not well understood yet. Chemical evolution models adopting different stellar yields can reproduce satisfactorily well the average trend of either $[\mathrm{Na} / \mathrm{Fe}]$ vs. $[\mathrm{Fe} / \mathrm{H}]$ or $[\mathrm{Al} / \mathrm{Fe}]$ vs. $[\mathrm{Fe} / \mathrm{H}]$ in the solar vicinity, but can never reproduce both simultaneously (see e.g. Romano et al. 2010; Nomoto et al. 2013, their Figs. 22 and 10 , respectively). Furthermore, the increase of $[\mathrm{Na} / \mathrm{Fe}]$ with metallicity observed for $[\mathrm{Fe} / \mathrm{H}]>0.00$ is not explained by the models.

\subsection{Trends with metallicity}

In Fig. 8, we show the predictions of model 15 of Romano et al. (2010); labeled Model A here, compared to those of other two models, obtained by assuming up-to-date prescriptions about stellar nucleosynthesis:

- Model A adopts the yields by Karakas (2010) for low- and intermediate-mass stars and the yields by Kobayashi et al. (2006) for massive stars; in particular, it assumes that all stars above $20 M_{\odot}$ explode as hypernovae, with energies much larger than normal supernovae;

- Model B is the same as Model A, but the yields for lowand intermediate-mass stars are from recent work published in Ventura et al. (2013, 2014a,b) and extend to super-solar metallicities;

- Model C is the same as Model B, but all massive stars explode as core-collapse supernovae with energies on the order of $10^{51} \mathrm{erg}$.

The model predictions are compared to measurements of $\mathrm{Na}$ and Al for dwarf stars in our sample (to avoid mixing effects on abundances) with high-quality data (dispersion below 0.15 dex and results based on four or more pipelines; see Sect. 2.3; large empty circles at $[\mathrm{Fe} / \mathrm{H}] \geq-1.0 \mathrm{dex}$ ); adding giants and lower quality data (small empty circles at $[\mathrm{Fe} / \mathrm{H}] \geq-1.5 \mathrm{dex}$ ) increases the dispersion, as expected. The data for the halo (turnoff and giant stars; small filled circles) are from Gehren et al. (2006), Andrievsky et al. (2007, for $\mathrm{Na}$ only) and Andrievsky et al. (2008, for Al only); to minimize spurious effects due to mass transfer from companions in binary systems or stellar evolution, we do not show the abundances of either known carbon-rich stars or mixed giants (i.e., stars located after the RGB bump; see Andrievsky et al. 2007, and references therein). All the ratios are normalized to the reference solar abundances adopted in this work (see Sect. 2.5). Furthermore, all $\mathrm{Na}$ abundances are corrected for non-LTE effects (see Sect. 2.6).

Notwithstanding the use of updated stellar yields, the detailed runs of $[\mathrm{Na} / \mathrm{Fe}]$ and $[\mathrm{Al} / \mathrm{Fe}]$ with $[\mathrm{Fe} / \mathrm{H}]$ in the solar vicinity remain largely unexplained. As expected (see Introduction), the contribution to $\mathrm{Na}$ and $\mathrm{Al}$ production from low- and intermediate-mass stars is negligible on a Galactic scale. The assumption that all stars above $20 M_{\odot}$ explode as hypernovae (Models $\mathrm{A}$ and $\mathrm{B}$ ) results in the lowest theoretical $[\mathrm{Al} / \mathrm{Fe}]$ ratios at the lowest metallicities, which is in reasonably good agreement with the observations, but also leads to extremely low $[\mathrm{Na} / \mathrm{Fe}]$ ratios that do not match $\mathrm{Na}$ observations for $[\mathrm{Fe} / \mathrm{H}]<$ -2.5. The model adopting the yields by Kobayashi et al. (2006) for normal core-collapse supernovae (Model C) does a better job for $\mathrm{Na}$ for $[\mathrm{Fe} / \mathrm{H}]<-2.5$, but severely overestimates the $[\mathrm{Al} / \mathrm{Fe}]$ ratios in the halo. Moreover, while it explains qualitatively the decreasing trend of $[\mathrm{Al} / \mathrm{Fe}]$ with metallicity for $[\mathrm{Fe} / \mathrm{H}]>-1.0$, it underproduces $\mathrm{Al}$ in the disk overall.

None of the models can explain the observed increase of $[\mathrm{Na} / \mathrm{Fe}]$ for $[\mathrm{Fe} / \mathrm{H}]>0$, which could suggest that the models lack some site of $\mathrm{Na}$ production at later stages. The missing source should be sufficiently strong to reverse the decreasing trend of $[\mathrm{Na} / \mathrm{Fe}]$ versus $[\mathrm{Fe} / \mathrm{H}]$ due to the delayed $\mathrm{Fe}$ production from supernovae ( $\mathrm{SNe}$ ) Ia. For this late $\mathrm{Na}$ production, we can think of two possible sites, i.e., SNe Ia and novae. The models shown here already account for some $\mathrm{Na}$ production from $\mathrm{SNe}$ Ia with yields from Iwamoto et al. (1999), but the contribution is negligible. Novae might also act in the right direction, since they restore the products of explosive $\mathrm{H}$ burning on relatively long timescales (see, e.g., Romano et al. 1999; Romano \& Matteucci 2003, and references therein). Indeed, novae have been shown to be able to contribute important amounts of ${ }^{7} \mathrm{Li},{ }^{13} \mathrm{C},{ }^{15} \mathrm{~N},{ }^{17} \mathrm{O}$, ${ }^{22} \mathrm{Na}$, and ${ }^{26} \mathrm{Al}$ (José \& Hernanz 1998, 2007), although the actual yields remain highly uncertain. It is worth remarking that Izzo et al. (2015) detected Li expelled by a nova system (see also 

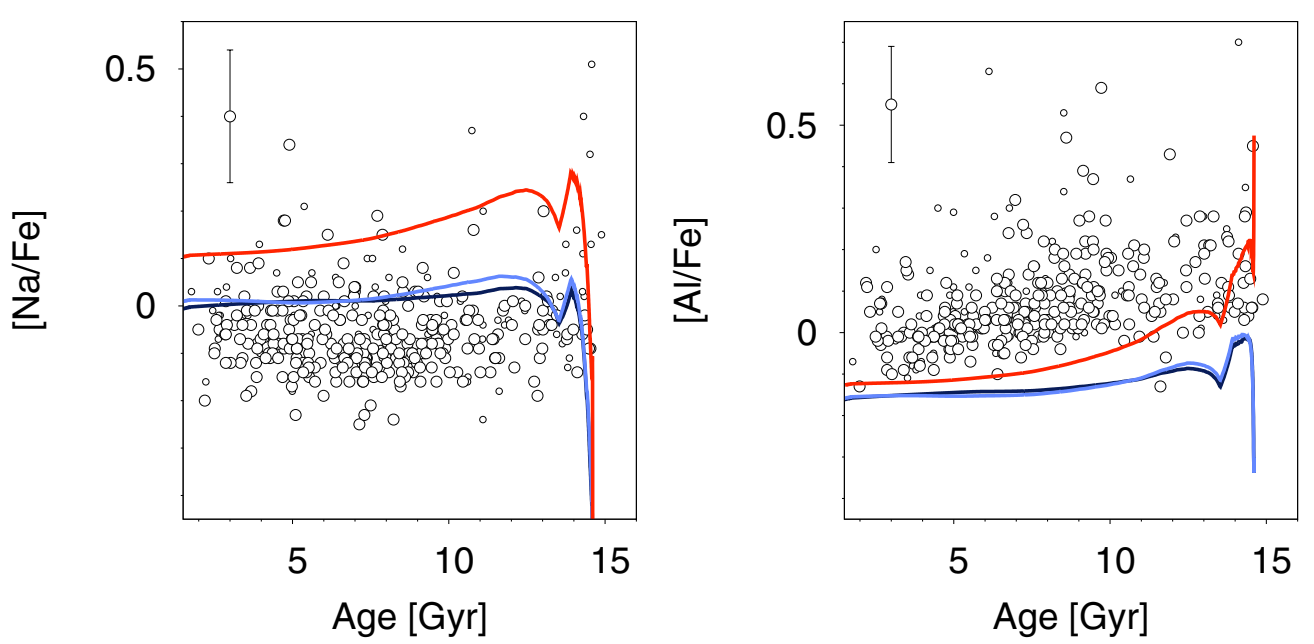

Fig. 9. Runs of $[\mathrm{Na} / \mathrm{Fe}]$ (left panel) and $[\mathrm{Al} / \mathrm{Fe}]$ (right panel) against age. Ages are available only for a subsample of Gaia-ESO solar neighborhood dwarfs with $[\mathrm{Fe} / \mathrm{H}]>-1.0$. Models and error bars are the same as in Fig. 8.
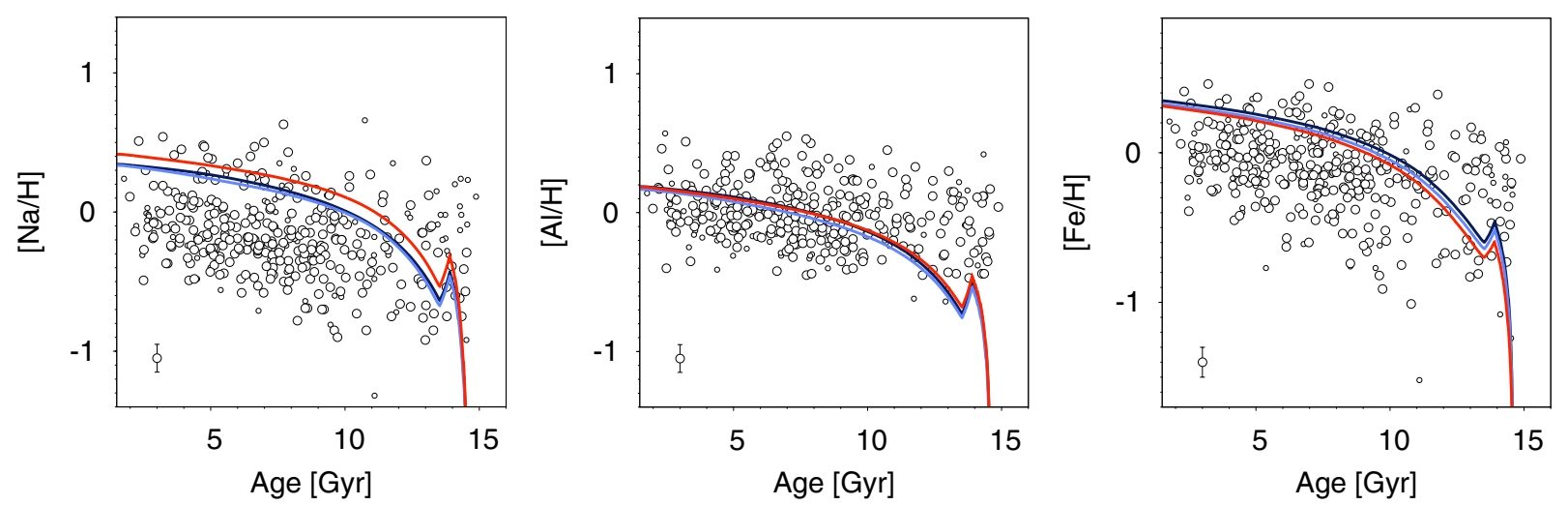

Fig. 10. Comparison of the observed ratios $[\mathrm{Na} / \mathrm{H}]$ (left panel), $[\mathrm{Al} / \mathrm{H}]($ central panel) and $[\mathrm{Fe} / \mathrm{H}]$ (right panel) and the model predictions as a function of age. Models and error bars are the same as in Fig. 8.

Tajitsu et al. 2015) and found a total amount of Li ejected in a single nova outburst that is significantly larger than expected from hydrodynamic nova models by José \& Hernanz (1998). In this context, it would be interesting to investigate whether some extra ${ }^{23} \mathrm{Na}$ can be produced in these same events in amounts that would reverse the decreasing trend of $[\mathrm{Na} / \mathrm{Fe}]$ with time predicted by current chemical evolution models (see next section). On the other hand, the possibility exists that the explanation of the increasing trend of $[\mathrm{Na} / \mathrm{Fe}]$ with time lies elsewhere. Indeed, $[\mathrm{Ni} / \mathrm{Fe}]$ also shows a clear upturn at $[\mathrm{Fe} / \mathrm{H}]>0$ (Bensby et al. 2014) which is hardly attributable to nova nucleosynthesis.

\subsection{Trends with age}

In Fig. 9, we show the plots of $[\mathrm{Na} / \mathrm{Fe}]$ (left panel) and $[\mathrm{Al} / \mathrm{Fe}]$ vs. age (right panel). Data are only available for a subset of the solar neighborhood Gaia-ESO dwarf sample with $[\mathrm{Fe} / \mathrm{H}] \geq-1.0$. The model predictions refer to the evolution of a thin-disk component (ages younger than 13.5 Gyr in Fig. 9), plus a thick-disk and halo component (ages older than 13.5 Gyr). Since the chemical evolution model assumes an age of the Universe of 13.7 Gyr and the procedure outlined in Sect. 2.7 leads to age estimates as old as $\sim 15 \mathrm{Gyr}$, we scaled the chemical evolution model results to an age of the Universe of 15 Gyr. In principle, comparing the observed and theoretical trends with age is quite instructive and might help to indicate why the models fail to reproduce the full behavior of the abundances.

Figure 9 shows that the observed trend of the $[\mathrm{Na} / \mathrm{Fe}]$ ratio in thin-disk stars is basically flat with respect to age. On the other hand, the models seem to predict (slightly) higher $[\mathrm{Na} / \mathrm{Fe}]$ values for older stars. It is worth stressing at this point that reliable stellar ages could be derived only for half of the original Gaia-ESO sample (see Sect. 2.7); therefore, our view of Na evolution could be biased in the abundance-versus-age diagram. Indeed, we notice that only a minority of the stars that have $[\mathrm{Na} / \mathrm{Fe}]>0.00$ in the $[\mathrm{Na} / \mathrm{Fe}]$ vs. $[\mathrm{Fe} / \mathrm{H}]$ plot (Fig. 8, left panel) is found in the $[\mathrm{Na} / \mathrm{Fe}]$ vs. age plot (Fig. 9, left panel). If most of the stars with $[\mathrm{Na} / \mathrm{Fe}]>0.00$ were young in age, the disagreement between the predicted and observed trends would worsen and this would strongly point to a missing late $\mathrm{Na}$ source. For $\mathrm{Al}$, in the solar neighborhood thin-disk stars there seems to be a weak trend of decreasing $[\mathrm{Al} / \mathrm{Fe}]$ for younger stars. As also seen in the trends with $[\mathrm{Fe} / \mathrm{H}]$, the models seem to predict consistently lower $[\mathrm{Al} / \mathrm{Fe}]$ than what is observed.

To separate the effects of the $\mathrm{Na}$ and $\mathrm{Al}$ evolution from that of Fe, we plot in Fig. 10 the trends of $[\mathrm{Na} / \mathrm{H}],[\mathrm{Al} / \mathrm{H}]$, and $[\mathrm{Fe} / \mathrm{H}]$ as a function of age (left, middle, and right panels, respectively). The models seem to predict an increasing trend for $[\mathrm{Na} / \mathrm{H}]$ that agrees with the observations but, again, we note that some Narich stars could be missing from the plot. Also, the increase in $[\mathrm{Fe} / \mathrm{H}]$ predicted by the model is a bit steeper than the increase 

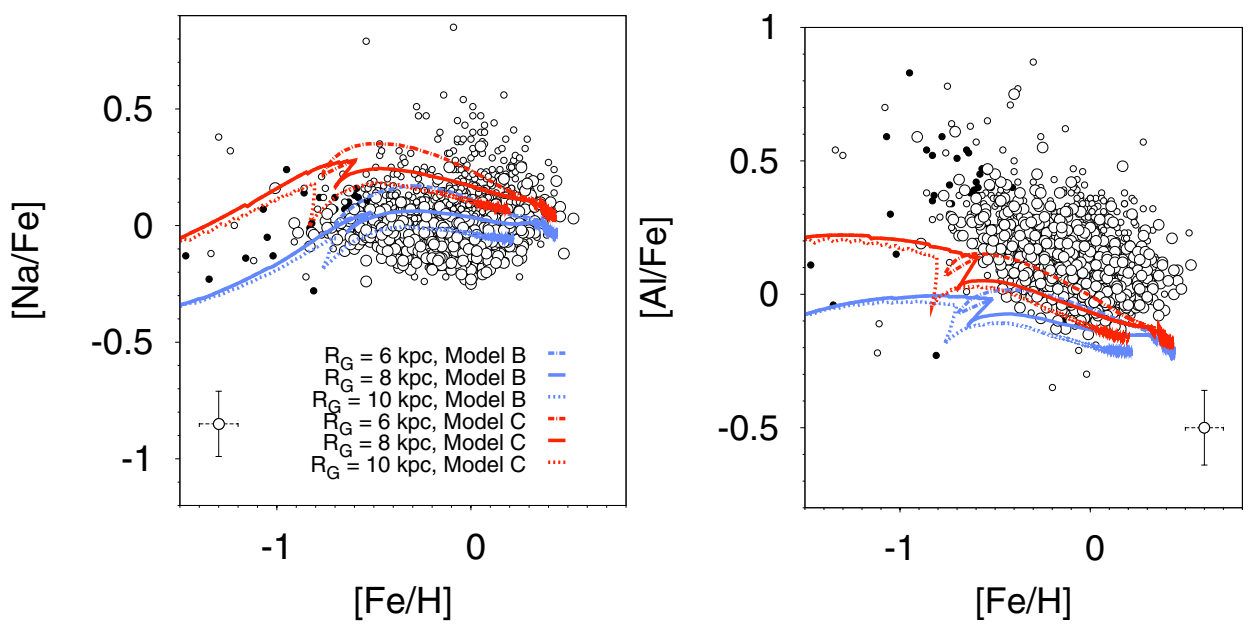

Fig. 11. Data for $[\mathrm{Na} / \mathrm{Fe}]$ vs. $[\mathrm{Fe} / \mathrm{H}]$ and $[\mathrm{Al} / \mathrm{Fe}]$ vs. $[\mathrm{Fe} / \mathrm{H}]$ (left and right panels, respectively) compared to the predictions of Models $\mathrm{B}$ and $\mathrm{C}$ run at $R_{\mathrm{G}}=6,8$, and $10 \mathrm{kpc}$. Error bars are the same as in Fig. 8 .

suggested by the observations. As for aluminium, there is a tendency of models to underproduce this element during the early disk evolution, while the predictions agree with the average observed trend of increasing $[\mathrm{Al} / \mathrm{H}]$ for ages younger than $7 \mathrm{Gyr}$ (Fig. 10, central panel). The stellar yields for this element clearly need to be revised as well.

\subsection{Trends with galactocentric distance}

It is well known that stars in a galactic disk can undergo important radial displacements and that these radial motions influence the chemical properties of the stellar populations (see Sellwood \& Binney 2002; Haywood 2008; Roškar et al. 2008; Schönrich \& Binney 2009; Minchev et al. 2013; Kubryk et al. 2013, among others). The migrating stars, in fact, coming from different Galactic regions, bear the imprints of different evolutionary rates. This has been put forward as a likely explanation for most of the observed spread in the age metallicity and $[\mathrm{El} / \mathrm{Fe}]$ vs. $[\mathrm{Fe} / \mathrm{H}]$ relations of solar neighborhood stars some 20 yr ago (Francois \& Matteucci 1993, and references therein).

Our subsample of Gaia-ESO solar neighborhood dwarfs with reasonable age estimates confirms previous findings, showing that the dispersion in $[\mathrm{Fe} / \mathrm{H}]$ values increases with increasing age. The situation for the ratios involving $\mathrm{Na}$ and $\mathrm{Al}$ is less clear (cf. Figs. 9 and 10). A detailed discussion of the amount and significance of the spreads is beyond the aim of this paper. However, in Fig. 11 we compare the $[\mathrm{Na} / \mathrm{Fe}]$ and $[\mathrm{Al} / \mathrm{Fe}]$ ratios of GaiaESO field dwarfs as functions of $[\mathrm{Fe} / \mathrm{H}]$ to the trends predicted by Models $\mathrm{B}$ and $\mathrm{C}$ (namely, with and without hypernovae, respectively) at different Galactic radii, $R_{\mathrm{G}}=6,8$, and $10 \mathrm{kpc}$. The models assume a star formation efficiency that varies with radius after Spitoni et al. (2015, see their Fig. 1). The dispersion in the abundance ratios could be explained, at least partly, by the radial migration of stars that formed at different radii and ended up in the solar vicinity. However, since we are using a pure chemical evolution model that does not include a detailed treatment of the stellar motions, we cannot make any quantitative prediction about the fractions of stars that are expected to be born at different radii.

\section{Summary}

We used new Na and Al abundances determined within the GaiaESO Survey, to readdress the behavior of these elements in what concerns both stellar and Galactic chemical evolution. For the stellar evolution discussion, we used a sample of giants in six open clusters, ranging in age from $300 \mathrm{Myr}$ to $4.5 \mathrm{Gyr}$. For the chemical evolution discussion, we used a sample of $\sim 600$ solar neighborhood dwarfs, complemented by halo stars from the literature. The Na abundances were corrected for non-LTE effects, and no corrections were applied to the $\mathrm{Al}$ abundances.

The average non-LTE $\mathrm{Na}$ abundances of the cluster giants show a trend of increasing $[\mathrm{Na} / \mathrm{Fe}]$ with increasing stellar mass, which is in agreement with expectations of stellar evolution models. Similar trends are seen in a selection of literature $\mathrm{Na}$ abundances of open cluster giants and in field giants with seismic masses derived thanks to CoRoT light curves. We consider the trend with mass as strong evidence of the stellar evolution origin of the surface $\mathrm{Na}$ enhancement seen in these giants. Nevertheless, for stars with mass below $\sim 2 M_{\odot}$, we cannot exclude a small $\mathrm{Na}$ enhancement, in disagreement with model predictions because of remaining uncertainties and possible systematics in the abundances. For stars with mass above $\sim 2 M_{\odot}$, we are not able to differentiate between models with and without rotation induced mixing.

Regarding $\mathrm{Al}$, no convincing evidence for a trend of $[\mathrm{Al} / \mathrm{Fe}]$ with stellar mass was found. Below $\sim 3 M_{\odot}$, the giants in our sample show a constant $\mathrm{Al}$ abundance. The only cluster with enhanced $\mathrm{Al}$ abundance, NGC 6705 with turn-off mass above $3 M_{\odot}$, has peculiar chemical composition. This suggests that its $\mathrm{Al}$ enhancement has origin in the environment where the cluster was formed (see Magrini et al. 2015).

The disagreement between Galactic chemical evolution models and observations for $\mathrm{Na}$ and $\mathrm{Al}$ in the solar neighborhood remains, even with the use of up-to-date stellar yields. The explanation for this disagreement does not seem to lie in lowand intermediate-mass stars, as their contribution to the increase in $\mathrm{Na}$ and $\mathrm{Al}$ in the Galaxy seems to be negligible. The average trend of $[\mathrm{Na} / \mathrm{Fe}]$ with $[\mathrm{Fe} / \mathrm{H}]$ in solar neighborhood dwarfs can be reproduced apart from the increase of $[\mathrm{Na} / \mathrm{Fe}]$ at higher metallicities. The observed and predicted trend of $[\mathrm{Na} / \mathrm{H}]$ with stellar age is, in principle, instructive to indicate where the problems seem to be. We note, however, that most of the stars with $[\mathrm{Na} / \mathrm{Fe}]>0.00$ have no reliable age determinations. This makes them disappear in the abundance vs. age diagram, which strongly affects any conclusion we might draw from this plot. Based on the $[\mathrm{Na} / \mathrm{Fe}]$ vs. $[\mathrm{Fe} / \mathrm{H}]$ diagram, we speculate that it is likely that some significant site of late Na production is missing from the models. 
The failure to reproduce the behavior of the $\mathrm{Al}$ abundances with metallicity and age is even more striking. For the solar neighborhood dwarfs with $[\mathrm{Fe} / \mathrm{H}]>-1.0, \mathrm{Al}$ is underproduced at all ages, but the youngest ones. For halo stars of lower metallicity, the models that nicely reproduce $\mathrm{Na}$ observations consistently overproduce Al. Clearly, a better understanding of the nucleosynthesis of $\mathrm{Al}$ is needed.

We will revisit the remaining open issues in our analysis when the Gaia-ESO survey is complete (it is currently in its fourth year of observations). On the stellar evolution side, further comparisons between low-mass dwarfs and giants in the same open clusters are needed to better understand possible differences in $\mathrm{Na}$ abundances. The increase in the sample of younger clusters will also facilitate a better discussion of $\mathrm{Na}$ and $\mathrm{Al}$ in giants with masses above $3 M_{\odot}$. On the chemical evolution side, we expect abundances with smaller uncertainties for a larger sample of solar neighborhood dwarfs and a new set of yields for massive stars computed considering the effects of stellar rotation on nucleosynthesis, from the pre-main sequence up to the explosive stages (M. Limongi, priv. comm.; and Chieffi \& Limongi 2015). This will provide tighter constraints on the evolution of $\mathrm{Na}$ and $\mathrm{Al}$ in the Galactic disk.

Acknowledgements. R.S. acknowledges support by the National Science Center of Poland through grant 2012/07/B/ST9/04428. T.M. acknowledges financial support from Belspo for contract PRODEX GAIA-DPAC. V.A. acknowledges the support from the Fundação para a Ciência e a Tecnologia (FCT) in the form of the grants SFRH/BPD/70574/2010 and PTDC/FIS-AST/1526/2014. G.T. acknowledges support by the grant from the Research Council of Lithuania (MIP082/2015). P.F. acknowledges support from the CNES. U.H. acknowledges support from the Swedish National Space Board (SNSB). Based on data products from observations made with ESO Telescopes at the La Silla Paranal Observatory under programme ID 188.B-3002. These data products have been processed by the Cambridge Astronomy Survey Unit (CASU) at the Institute of Astronomy, University of Cambridge, and by the FLAMES/UVES reduction team at INAF/Osservatorio Astrofisico di Arcetri. These data have been obtained from the Gaia-ESO Survey Data Archive, prepared and hosted by the Wide Field Astronomy Unit, Institute for Astronomy, University of Edinburgh, which is funded by the UK Science and Technology Facilities Council. This work was partly supported by the European Union FP7 programme through ERC grant number 320360 and by the Leverhulme Trust through grant RPG-2012541. We acknowledge the support from INAF and Ministero dell' Istruzione, dell' Universita' e della Ricerca (MIUR) in the form of the grant "Premiale VLT 2012" and the grant "The Chemical and Dynamical Evolution of the Milky Way and Local Group Galaxies" (prot. 2010LY5N2T). The results presented here benefit from discussions held during the Gaia-ESO workshops and conferences supported by the ESF (European Science Foundation) through the GREAT Research Network Programme. This research has made use of the SIMBAD database, operated at CDS, Strasbourg, France, NASA's Astrophysics Data System, and the WEBDA database, operated at the Department of Theoretical Physics and Astrophysics of Masaryk University.

\section{References}

Adibekyan, V. Z., Sousa, S. G., Santos, N. C., et al. 2012, A\&A, 545, A32 Adibekyan, V. Z., Benamati, L., Santos, N. C., et al. 2015, MNRAS, 450, 1900 Alexeeva, S. A., Pakhomov, Y. V., \& Mashonkina, L. I. 2014, Astron. Lett., 40, 406

Andrievsky, S. M., Egorova, I. A., Korotin, S. A., \& Burnage, R. 2002, A\&A, 389, 519

Andrievsky, S. M., Spite, M., Korotin, S. A., et al. 2007, A\&A, 464, 1081 Andrievsky, S. M., Spite, M., Korotin, S. A., et al. 2008, A\&A, 481, 481 Arnett, W. D., \& Thielemann, F.-K. 1985, ApJ, 295, 589

Aurière, M. 2003, in EAS PS 9, eds. J. Arnaud, \& N. Meunier, 105

Baglin, A., Auvergne, M., Boisnard, L., et al. 2006, in COSPAR Meeting, 36, 36th COSPAR Scientific Assembly, 3749

Baudin, F., Barban, C., Goupil, M. J., et al. 2012, A\&A, 538, A73

Baumueller, D., \& Gehren, T. 1996, A\&A, 307, 961

Baumueller, D., \& Gehren, T. 1997, A\&A, 325, 1088

Beaver, J., Kaltcheva, N., Briley, M., \& Piehl, D. 2013, PASP, 125, 1412
Belyaev, A. K. 2013, A\&A, 560, A60

Bensby, T., Feltzing, S., \& Oey, M. S. 2014, A\&A, 562, A71

Bergemann, M., Ruchti, G. R., Serenelli, A., et al. 2014, A\&A, 565, A89

Blanco-Cuaresma, S., Soubiran, C., Jofré, P., \& Heiter, U. 2014, A\&A, 566, A98

Boesgaard, A. M., Lum, M. G., \& Deliyannis, C. P. 2015, ApJ, 799, 202

Borucki, W. J., Koch, D., Basri, G., et al. 2010, Science, 327, 977

Bragaglia, A., \& Tosi, M. 2006, AJ, 131, 1544

Bragaglia, A., Gratton, R. G., Carretta, E., et al. 2012, A\&A, 548, A122

Bragaglia, A., Sneden, C., Carretta, E., et al. 2014, ApJ, 796, 68

Bressan, A., Marigo, P., Girardi, L., et al. 2012, MNRAS, 427, 127

Cameron, A. G. W. 1959, ApJ, 130, 429

Cantat-Gaudin, T., Vallenari, A., Zaggia, S., et al. 2014, A\&A, 569, A17

Carraro, G., \& Ortolani, S. 1994, A\&AS, 106, 573

Carraro, G., Geisler, D., Moitinho, A., Baume, G., \& Vázquez, R. A. 2005, A\&A, 442, 917

Carraro, G., Geisler, D., Villanova, S., Frinchaboy, P. M., \& Majewski, S. R. 2007, A\&A, 476, 217

Carraro, G., Villanova, S., Monaco, L., et al. 2014, A\&A, 562, A39

Carrera, R., \& Pancino, E. 2011, A\&A, 535, A30

Carretta, E., Gratton, R. G., \& Sneden, C. 2000, A\&A, 356, 238

Carretta, E., Bragaglia, A., Gratton, R. G., \& Tosi, M. 2005, A\&A, 441, 131

Cayrel, R., Depagne, E., Spite, M., et al. 2004, A\&A, 416, 1117

Charbonnel, C., \& Lagarde, N. 2010, A\&A, 522, A10

Chieffi, A., \& Limongi, M. 2015, in IAU Symp. 307, eds. G. Meynet, C. Georgy, J. Groh, \& P. Stee, 1

Collet, R., Asplund, M., \& Trampedach, R. 2007, A\&A, 469, 687

Cunha, K., Smith, V. V., Johnson, J. A., et al. 2015, ApJ, 798, L41

Dekker, H., D’Odorico, S., Kaufer, A., Delabre, B., \& Kotzlowski, H. 2000, in Optical and IR Telescope Instrumentation and Detectors, eds. M. Iye, \& A. F. Moorwood, SPIE Conf. Ser., 4008, 534

Denissenkov, P. A. 2005, ApJ, 622, 1058

Denisenkov, P. A., \& Denisenkova, S. N. 1990, Sov. Astron. Lett., 16, 275

Dobrovolskas, V., Kučinskas, A., Steffen, M., et al. 2013, A\&A, 559, A102

Doherty, C. L., Gil-Pons, P., Lau, H. H. B., Lattanzio, J. C., \& Siess, L. 2014, MNRAS, 437, 195

Donati, P., Beccari, G., Bragaglia, A., Cignoni, M., \& Tosi, M. 2014a, MNRAS, 437,1241

Donati, P., Cantat Gaudin, T., Bragaglia, A., et al. 2014b, A\&A, 561, A94

Edvardsson, B., Andersen, J., Gustafsson, B., et al. 1993, A\&A, 275, 101

El Eid, M. F., \& Champagne, A. E. 1995, ApJ, 451, 298

François, P. 1986a, A\&A, 160, 264

François, P. 1986b, A\&A, 165, 183

Francois, P., \& Matteucci, F. 1993, A\&A, 280, 136

François, P., Pasquini, L., Biazzo, K., Bonifacio, P., \& Palsa, R. 2013, A\&A, 552, A136

Friel, E. D., Jacobson, H. R., Barrett, E., et al. 2003, AJ, 126, 2372

Friel, E. D., Donati, P., Bragaglia, A., et al. 2014, A\&A, 563, A117

Gehren, T., Liang, Y. C., Shi, J. R., Zhang, H. W., \& Zhao, G. 2004, A\&A, 413, 1045

Gehren, T., Shi, J. R., Zhang, H. W., Zhao, G., \& Korn, A. J. 2006, A\&A, 451, 1065

Geisler, D., Villanova, S., Carraro, G., et al. 2012, ApJ, 756, L40

Gilmore, G., Randich, S., Asplund, M., et al. 2012, The Messenger, 147, 25

Gratton, R. G., Carretta, E., Eriksson, K., \& Gustafsson, B. 1999, A\&A, 350, 955

Gratton, R. G., Sneden, C., Carretta, E., \& Bragaglia, A. 2000, A\&A, 354, 169

Gratton, R. G., Carretta, E., \& Bragaglia, A. 2012, A\&ARv, 20, 50

Grevesse, N., Asplund, M., \& Sauval, A. J. 2007, Space Sci. Rev., 130, 105

Hayes, C. R., \& Friel, E. D. 2014, AJ, 147, 69

Haywood, M. 2008, MNRAS, 388, 1175

Heiter, U., Jofré, P., Gustafsson, B., et al. 2015a, A\&A, 582, A49

Heiter, U., Lind, K., Asplund, M., et al. 2015b, Phys. Scr., 90, 054010

Helfer, H. L., \& Wallerstein, G. 1964, ApJS, 9, 81

Iben, Jr., I. 1964, ApJ, 140, 1631

Iben, Jr., I. 1967, ARA\&A, 5, 571

Iwamoto, K., Brachwitz, F., Nomoto, K., et al. 1999, ApJS, 125, 439

Izzo, L., Della Valle, M., Mason, E., et al. 2015, ApJ, 808, L14

Jacobson, H. R., Friel, E. D., \& Pilachowski, C. A. 2007, AJ, 134, 1216

Jofré, P., Heiter, U., Soubiran, C., et al. 2014, A\&A, 564, A133

José, J., \& Hernanz, M. 1998, ApJ, 494, 680

José, J., \& Hernanz, M. 2007, J. Phys. G Nucl. Phys., 34, 431

Kallinger, T., Weiss, W. W., Barban, C., et al. 2010, A\&A, 509, A77

Kaluzny, J., Krzeminski, W., \& Mazur, B. 1996, A\&AS, 118, 303

Karakas, A. I. 2010, MNRAS, 403, 1413

Kaulakys, B. 1991, J. Phys. B, 24, L127

Kobayashi, C., Umeda, H., Nomoto, K., Tominaga, N., \& Ohkubo, T. 2006, ApJ, 653,1145

Kordopatis, G., Wyse, R. F. G., Gilmore, G., et al. 2015, A\&A, 582, A122 
Kovtyukh, V. V., Wallerstein, G., \& Andrievsky, S. M. 2005, PASP, 117, 1173 Kubryk, M., Prantzos, N., \& Athanassoula, E. 2013, MNRAS, 436, 1479 Lagarde, N., Decressin, T., Charbonnel, C., et al. 2012, A\&A, 543, A108 Lagarde, N., Anderson, R. I., Charbonnel, C., et al. 2014, A\&A, 570, C2 Lagarde, N., Miglio, A., Eggenberger, P., et al. 2015, A\&A, 580, A141 Lanzafame, A. C., Frasca, A., Damiani, F., et al. 2015, A\&A, 576, A80 Lind, K., Asplund, M., Barklem, P. S., \& Belyaev, A. K. 2011, A\&A, 528, A103 Luck, R. E., \& Heiter, U. 2006, AJ, 131, 3069

MacLean, B. T., De Silva, G. M., \& Lattanzio, J. 2015, MNRAS, 446, 3556

Magrini, L., Randich, S., Romano, D., et al. 2014, A\&A, 563, A44

Magrini, L., Randich, S., Donati, P., et al. 2015, A\&A, 580, A85

McWilliam, A., Preston, G. W., Sneden, C., \& Searle, L. 1995, AJ, 109, 2757

Mikolaitis, Š., Tautvaišienè, G., Gratton, R., Bragaglia, A., \& Carretta, E. 2010, MNRAS, 407, 1866

Mikolaitis, Š., Tautvaišienè, G., Gratton, R., Bragaglia, A., \& Carretta, E. 2011, MNRAS, 413, 2199

Mikolaitis, Š., Hill, V., Recio-Blanco, A., et al. 2014, A\&A, 572, A33

Minchev, I., Chiappini, C., \& Martig, M. 2013, A\&A, 558, A9

Mishenina, T. V., Bienaymé, O., Gorbaneva, T. I., et al. 2006, A\&A, 456, 1109

Mishenina, T. V., Soubiran, C., Bienaymé, O., et al. 2008, A\&A, 489, 923

Morel, T., Miglio, A., Lagarde, N., et al. 2014, A\&A, 564, A119

Mosser, B., Belkacem, K., Goupil, M.-J., et al. 2010, A\&A, 517, A22

Mowlavi, N. 1999, A\&A, 350, 73

Nomoto, K., Kobayashi, C., \& Tominaga, N. 2013, ARA\&A, 51, 457

Pancino, E., Carrera, R., Rossetti, E., \& Gallart, C. 2010, A\&A, 511, A56

Pasquini, L., Avila, G., Blecha, A., et al. 2002, The Messenger, 110, 1

Peterson, R. C. 1981, ApJ, 244, 989

Pilachowski, C. A., Sneden, C., \& Kraft, R. P. 1996, AJ, 111, 1689

Randich, S., \& Gilmore, G. 2013, The Messenger, 154, 47

Recio-Blanco, A., de Laverny, P., Kordopatis, G., et al. 2014, A\&A, 567, A5

Reddy, B. E., Lambert, D. L., \& Allende Prieto, C. 2006, MNRAS, 367, 1329

Romano, D., \& Matteucci, F. 2003, MNRAS, 342, 185

Romano, D., Matteucci, F., Molaro, P., \& Bonifacio, P. 1999, A\&A, 352, 117

Romano, D., Karakas, A. I., Tosi, M., \& Matteucci, F. 2010, A\&A, 522, A32

Roškar, R., Debattista, V. P., Quinn, T. R., Stinson, G. S., \& Wadsley, J. 2008, ApJ, 684, L79

Sacco, G. G., Morbidelli, L., Franciosini, E., et al. 2014, A\&A, 565, A113

Salpeter, E. E. 1952, ApJ, 115, 326

Salpeter, E. E. 1955, Phys. Rev., 97, 1237

Santrich, O. J. K., Pereira, C. B., \& Drake, N. A. 2013, A\&A, 554, A2

Schönrich, R., \& Binney, J. 2009, MNRAS, 396, 203

Schuler, S. C., King, J. R., \& The, L.-S. 2009, ApJ, 701, 837

Seleznev, A. F., Carraro, G., Costa, E., \& Loktin, A. V. 2010, New Astron., 15, 61

Sellwood, J. A., \& Binney, J. J. 2002, MNRAS, 336, 785

Serenelli, A. M., Bergemann, M., Ruchti, G., \& Casagrande, L. 2013, MNRAS, 429, 3645

Sestito, P., Bragaglia, A., Randich, S., et al. 2008, A\&A, 488, 943

Smiljanic, R. 2012, MNRAS, 422, 1562

Smiljanic, R., Gauderon, R., North, P., et al. 2009, A\&A, 502, 267

Smiljanic, R., Korn, A. J., Bergemann, M., et al. 2014, A\&A, 570, A122

Spite, M., \& Spite, F. 1980, A\&A, 89, 118

Spitoni, E., Romano, D., Matteucci, F., \& Ciotti, L. 2015, ApJ, 802, 129

Stello, D., Bruntt, H., Preston, H., \& Buzasi, D. 2008, ApJ, 674, L53

Tajitsu, A., Sadakane, K., Naito, H., Arai, A., \& Aoki, W. 2015, Nature, 518, 381

Takeda, Y., \& Takada-Hidai, M. 1994, PASJ, 46, 395

Takeda, Y., Kang, D.-I., Han, I., Lee, B.-C., \& Kim, K.-M. 2013, MNRAS, 432, 769

Tautvaišienė, G., Edvardsson, B., Tuominen, I., \& Ilyin, I. 2000, A\&A, 360, 499

Tautvaišienė, G., Edvardsson, B., Puzeras, E., \& Ilyin, I. 2005, A\&A, 431, 933

Tautvaišienė, G., Drazdauskas, A., Mikolaitis, Š., et al. 2015, A\&A, 573, A55

Thielemann, F. K., \& Arnett, W. D. 1985, ApJ, 295, 604

Ventura, P., Di Criscienzo, M., Carini, R., \& D’Antona, F. 2013, MNRAS, 431, 3642

Ventura, P., Criscienzo, M. D., D’Antona, F., et al. 2014a, MNRAS, 437, 3274

Ventura, P., Dell'Agli, F., Schneider, R., et al. 2014b, MNRAS, 439, 977

Villanova, S., Carraro, G., \& Saviane, I. 2009, A\&A, 504, 845

Wallerstein, G. 1962, ApJS, 6, 407

Weiss, A., \& Charbonnel, C. 2004, Mem. Soc. Astron. It., 75, 347
Weiss, A., \& Schlattl, H. 2008, Ap\&SS, 316, 99

Woosley, S. E., \& Weaver, T. A. 1995, ApJS, 101, 181

Yong, D., Carney, B. W., \& Friel, E. D. 2012, AJ, 144, 95

1 Department for Astrophysics, Nicolaus Copernicus Astronomical Center, ul. Rabiańska 8, 87-100 Toruń, Poland e-mail: rsmiljanic@ncac.torun.pl

2 INAF-Osservatorio Astronomico di Bologna, via Ranzani 1, 40127 Bologna, Italy

3 Dipartimento di Fisica e Astronomia, Università di Bologna, via Ranzani 1, 40127 Bologna, Italy

4 INAF-Osservatorio Astrofisico di Arcetri, Largo Enrico Fermi 5 50125 Firenze, Italy

5 Department of Astronomy, Indiana University, Bloomington, IN 47405, USA

6 Kavli Institute for Astrophysics \& Space Research, Massachusetts Institute of Technology, 77 Massachusetts Avenue, Cambridge, MA 02139, USA

7 INAF-Osservatorio Astronomico di Roma, via Frascati 33, 00040 Monte Porzio Catone (RM), Italy

8 Department of Physics and Astronomy, Uppsala University, Box 516, 75120 Uppsala, Sweden

9 Max Planck Institute für Astronomy, Königstuhl 17, 69117 Heidelberg, Germany

10 Institut d'Astrophysique et de Géophysique, Université de Liège, Allée du 6 Août, Bât. B5c, 4000 Liège, Belgium

11 ASI Science Data Center, via del Politecnico SNC, 00133 Roma, Italy

12 Institute of Theoretical Physics and Astronomy, Vilnius University, Goštauto 12, 01108 Vilnius, Lithuania

13 Instituto de Astrofísica e Ciências do Espaço, Universidade do Porto, CAUP, Rua das Estrelas, 4150-762 Porto, Portugal

14 INAF-Osservatorio Astronomico di Padova, Vicolo Osservatorio 2, 35122 Padova, Italy

15 Institute of Astronomy, University of Cambridge, Madingley Road, Cambridge CB3 0HA, UK

16 Lund Observatory, Department of Astronomy and Theoretical Physics, Box 43, 22100 Lund, Sweden

17 GEPI, Observatoire de Paris, PSL Research University, CNRS, Univ. Paris Diderot, Sorbonne Paris Cité, 61 avenue de l'Observatoire, 75014 Paris, France

18 Université de Picardie Jules Verne, Physics Dpt. 33 rue St. Leu, 80000 Amiens, France

19 Dipartimento di Fisica e Astronomia, Sezione Astrofisica, Universitá di Catania, via S. Sofia 78, 95123 Catania, Italy

20 Laboratoire Lagrange (UMR7293), Université de Nice Sophia Antipolis, CNRS, Observatoire de la Côte d'Azur, CS 34229, 06304 Nice Cedex 4, France

21 Instituto de Física y Astronomía, Universidad de Valparaíso, Chile

22 European Southern Observatory, Alonso de Cordova 3107 Vitacura, Santiago de Chile, Chile

23 Instituto de Astrofísica de Andalucía-CSIC, Apdo. 3004, 18080 Granada, Spain

24 Department of Physics and Astronomy, Uppsala University, Box 516, 75120 Uppsala, Sweden

25 Astrophysics Research Institute, Liverpool John Moores University, 146 Brownlow Hill, Liverpool L3 5RF, UK

26 Departamento de Ciencias Fisicas, Universidad Andres Bello, Republica 220, Santiago, Chile

27 Millennium Institute of Astrophysics, Av. Vicuña Mackenna 4860, 782-0436 Macul, Santiago, Chile

28 Pontificia Universidad Católica de Chile, Av. Vicuña Mackenna 4860, 782-0436 Macul, Santiago, Chile 